Radiative neutron capture as a counting technique at pulsed spallation neutron sources: a review of current progress

This content has been downloaded from IOPscience. Please scroll down to see the full text. 2016 Rep. Prog. Phys. 79094301

(http://iopscience.iop.org/0034-4885/79/9/094301)

View the table of contents for this issue, or go to the journal homepage for more

Download details:

IP Address: 150.146.205.185

This content was downloaded on 16/08/2016 at 10:36

Please note that terms and conditions apply. 


\title{
Radiative neutron capture as a counting technique at pulsed spallation neutron sources: a review of current progress
}

\author{
E M Schooneveld ${ }^{1}$, A Pietropaolo ${ }^{2,3}$, C Andreani $^{4}$, E Perelli Cippo ${ }^{5}$, \\ N J Rhodes ${ }^{1}$, R Senesi ${ }^{3}$, M Tardocchi ${ }^{5}$ and G Gorini ${ }^{6}$ \\ 1 Science and Technology Facilities Council, Didcot, Oxfordshire, UK \\ 2 ENEA Centro Ricerche Frascati, Via E. Fermi 45, I-00044 Frascati, Rome, Italy \\ 3 MIFP-Mediterranean Institute of Fundamental Physics, Via Appia Nuova 3100040 Marino, Rome, Italy \\ ${ }^{4}$ Department of Physics and NAST Centre, Universita' degli Studi di Roma Tor Vergata, \\ Via della Ricerca Scientifica 1, 00133 Roma and CNR-IPCF sezione di Messina and Centro Fermi, Italy \\ 5 Istituto di Fisica del Plasma 'Piero Caldirola', CNR, via Cozzi 53, I-20125 Milano, Italy \\ ${ }^{6}$ Universita Milano Bicocca, Piazza della Scienza 3, 20126 Milano, Italy
}

E-mail: erik.schooneveld@stfc.ac.uk, antonino.pietropaolo@enea.it, carla.andreani@uniroma2.it, perelli@ifp.cnr.it,nigel.rhodes@stfc.ac.uk,roberto.senesi@uniroma2.it, tardocchi@ifp.cnr.it and giuseppe.gorini@mib.infn.it

Received 13 May 2015, revised 15 April 2016

Accepted for publication 18 April 2016

Published 9 August 2016

\begin{abstract}
Neutron scattering techniques are attracting an increasing interest from scientists in various research fields, ranging from physics and chemistry to biology and archaeometry. The success of these neutron scattering applications is stimulated by the development of higher performance instrumentation. The development of new techniques and concepts, including radiative capture based neutron detection, is therefore a key issue to be addressed.

Radiative capture based neutron detectors utilize the emission of prompt gamma rays after neutron absorption in a suitable isotope and the detection of those gammas by a photon counter. They can be used as simple counters in the thermal region and (simultaneously) as energy selector and counters for neutrons in the eV energy region. Several years of extensive development have made $\mathrm{eV}$ neutron spectrometers operating in the so-called resonance detector spectrometer (RDS) configuration outperform their conventional counterparts. In fact, the VESUVIO spectrometer, a flagship instrument at ISIS serving a continuous user programme for $\mathrm{eV}$ inelastic neutron spectroscopy measurements, is operating in the RDS configuration since 2007.

In this review, we discuss the physical mechanism underlying the RDS configuration and the development of associated instrumentation. A few successful neutron scattering experiments that utilize the radiative capture counting techniques will be presented together with the potential of this technique for thermal neutron diffraction measurements. We also outline possible improvements and future perspectives for radiative capture based neutron detectors in neutron scattering application at pulsed neutron sources.
\end{abstract}

Keywords: neutron detectors, radiative capture, spallation neutron sources

(Some figures may appear in colour only in the online journal) 


\section{Introduction}

Neutron-based techniques are gaining an increasing interest from a continuously growing community of scientists in different research fields, ranging from physics and chemistry to biology and archaeometry. This mostly relies upon the unique properties of the neutron: being a neutral particle, it interacts weakly with materials and thus it is capable of probing bulky samples. Neutron scattering techniques are able to provide information on structure and dynamics of condensed matter. 'Where atoms are' and 'how atoms move' was the great achievement of Shull and Brockhouse using thermal neutron scattering [1-4]. Since that period these techniques were continuously refined and strengthened, extending the horizons of scientists working in different fields. The advent of spallation neutron sources [5] offered the possibility to further enhance the great potential of neutron scattering techniques: new techniques were born thanks to the availability of intense fluxes of neutrons above the thermal region, such as the neutron Compton scattering [6-16] or inelastic neutron-electron scattering [17-20].

The potential envisaged by means of theoretical speculations could be realized because of the continuous development of dedicated instrumentation. In turn, the development of new and high performance instrumentation opened the way to new experimental investigations that inspired theoretical approaches, in a sort of synergic loop. As a matter of fact, the neutron detection techniques are a key issue to be addressed for the improvement of instrument capabilities. In this particular context, the recent problem of ${ }^{3} \mathrm{He}$ shortage stimulates and inspires research and development activities on thermal neutron detection approaches. In the case of epithermal neutrons, standard detectors based on ${ }^{6} \mathrm{Li}$ or ${ }^{3} \mathrm{He}$ nuclei have a low detection efficiency, due to the $1 / v$ dependence of their neutron absorption cross section, $v$ being the neutron velocity. In this review, an overview is presented of a neutron detection approach based on the $(n, \gamma)$ conversion reactions for both thermal and epithermal neutron scattering at spallation neutron sources. The main focus will be on epithermal neutrons as the detection technique based on $(n, \gamma)$ reactions reached its maturity, while thermal neutron radiative capture is at its early, although promising, stage for time of flight neutron diffraction.

\section{Neutron radiative capture reactions}

Radiative capture relies on the absorption of a neutron by a nucleus followed by the prompt $\left(\tau \simeq 10^{-14} \mathrm{~s}\right)$ emission of gamma rays. The capture reaction can be modelled as the formation and decay of a 'compound nucleus' following the reaction $[21,22]$ :

$$
{ }^{A} X_{Z}+n \rightarrow\left({ }^{A+1} X_{Z}\right)^{*} \rightarrow{ }^{A+1} X_{Z}+\gamma+Q .
$$

The $Q$-value of the radiative capture reaction can be written, given the zero photon rest mass and indicating the neutron mass with $m_{n}$, as:

$$
\left[\left(M\left({ }^{A} X_{Z}\right)+m_{n}\right)-M\left({ }^{A+1} X_{Z}\right)\right]
$$

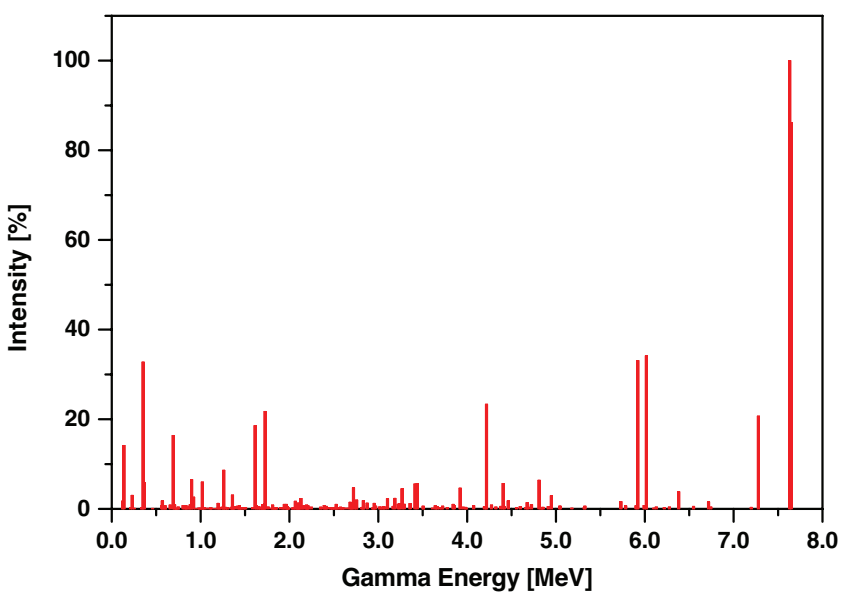

Figure 1. Capture gamma ray spectrum for ${ }^{56} \mathrm{Fe}$. The direct transition to the ground state dominates de-excitation of the nucleus, as the most intense gamma emission is at the highest gamma energy. Data taken from [23].

which is the energy needed to separate a neutron from the compound nucleus. Since neutron separation energies are always positive, radiative capture is an exoergic reaction: the threshold energy is zero, so that neutrons of any energy may trigger the reaction. Upon neutron capture, the compound nucleus is likely found at an excited level in turn decaying by the emission of one or more gamma rays to reach the ground state. Neglecting the recoil energy of the nucleus upon gamma emission, the total amount of energy produced by the radiative process is equal to the de-excitation energy. In what follows, the radiative capture reactions for thermal and epithermal neutrons are briefly described.

\subsection{Thermal neutron radiative capture}

Radiative capture gamma ray spectra resulting from thermal neutron capture have been measured for many isotopes and recorded over many years [23]. The decay mode of the compound nucleus upon thermal neutron capture is specific to the absorbing system. In this respect, three major types of capture gamma ray spectra can be identified. The first type are spectra characterized by a few emission lines where the direct transition from the absorbing level to the ground state dominates. In these cases, most of the energy is carried by a single gamma ray of energy in the order of 6-8 MeV; see for example figure 1 showing the radiative capture gamma ray spectrum for ${ }^{56} \mathrm{Fe}$. The second type of spectra are featuring a resolved line structure, such as that of light and medium weight elements with large spacing among single particle state levels and where the transition to intermediate levels are as likely as direct ground state transitions. The third type of spectra has many unresolved lines. For these spectra, especially those of heavy elements that have a high level density, the transition to any level can be well described by statistical approaches [21, 22]. It is found that the number of gamma rays in the neutron captureinduced spectrum depends on the number of states below the excited state of the nucleus after neutron absorption. In fact, in the case of ${ }^{1} \mathrm{H}$, the ${ }^{2} \mathrm{H}$ nucleus features only two states and the corresponding gamma spectrum is composed of only one 


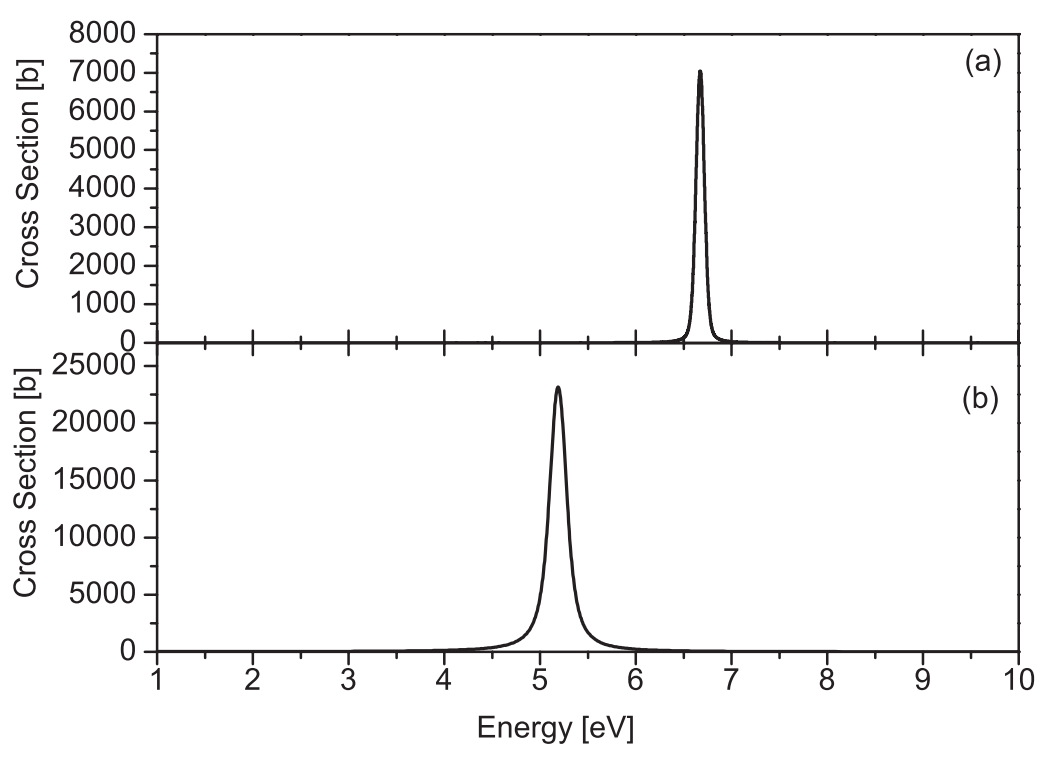

Figure 2. Radiative capture cross section of (a) ${ }^{238} \mathrm{U}$ and (b) ${ }^{207} \mathrm{Ag}$ in the epithermal neutron energy range below $10 \mathrm{eV}$.

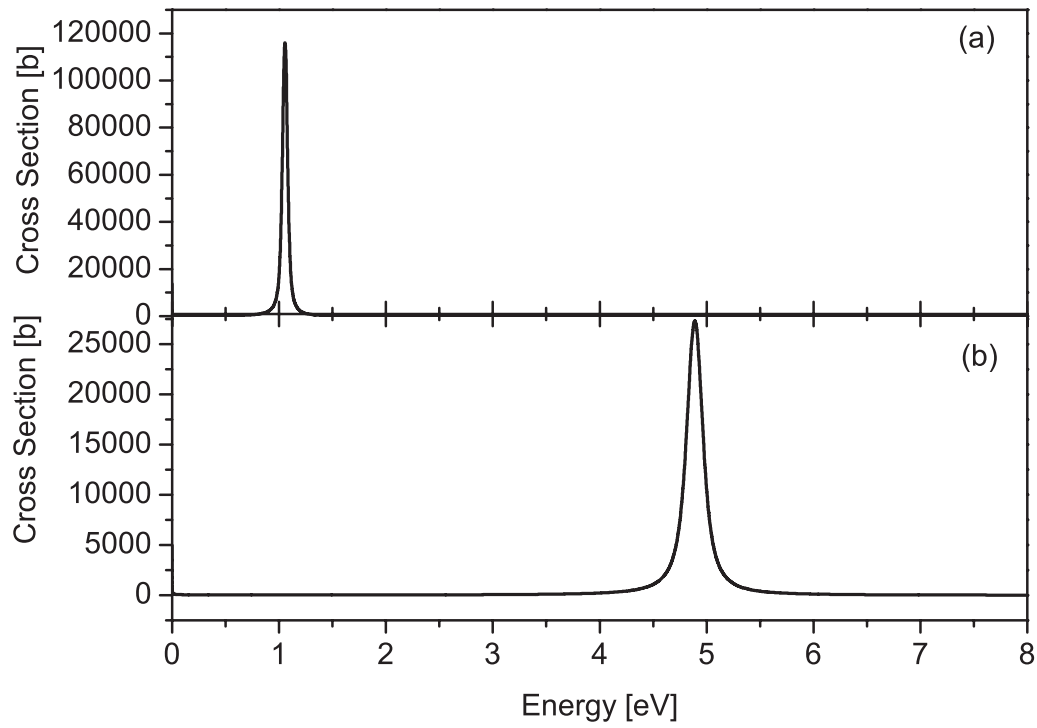

Figure 3. Radiative capture cross section of (a) ${ }^{240} \mathrm{Pu}$ and (b) ${ }^{197} \mathrm{Au}$ in the epithermal neutron energy range below $10 \mathrm{eV}$.

line at $2.22 \mathrm{MeV}$, i.e. binding energy of one neutron in a ${ }^{2} \mathrm{H}$ nucleus. For a thorough and more exhaustive discussion of these issues the reader is referred to [21-23].

\subsection{Resonance neutron radiative capture}

For low energy neutrons, and energies below the first capture resonance, the radiative capture cross section $\sigma_{(n, \gamma)}$ is found to vary as $1 / v$, with $v$ being the neutron velocity. In the proximity of an isolated resonance, the radiative capture cross section is well described by the Breit-Wigner line shape [24]. In medium to heavy nuclei the isolated resonances are found at low energies as indicated, for example, in figure 2 for the case of ${ }^{238} \mathrm{U}$ and ${ }^{207} \mathrm{Ag}$, data from [25]. In this case, the only term that contributes to the partial wave expansion of the interaction cross section is that with angular momentum $l=0$ and the $\sigma_{(n, \gamma)}$ can be written as:

$$
\sigma_{(n, \gamma)}(E)=\frac{\lambda^{2} \cdot(2 J+1)}{8 \pi^{3} \cdot(2 I+1)} \frac{\Gamma_{n} \Gamma_{\gamma}}{\left(E-E_{\mathrm{r}}\right)^{2}+\frac{\Gamma^{2}}{4}}
$$

where $I$ is the spin of the target nucleus, $J$ that of the compound nucleus, $E_{\mathrm{r}}$ the resonance energy and $\Gamma_{n}$ and $\Gamma_{\gamma}$ the partial widths for neutron and gamma emission, with $\Gamma=\Gamma_{n}+\Gamma_{\gamma}$ being the total width. The radiative capture width, $\Gamma_{\gamma}$, is typically on the order of $0.1-0.5 \mathrm{eV}$ for medium mass nuclei and is somewhat smaller, around $0.1 \mathrm{eV}$ or less, for large mass nuclei. Figure 3 reports the resonance cross section for two different nuclear masses, namely ${ }^{240} \mathrm{Pu}$ and ${ }^{197} \mathrm{Au}$. The partial gamma width, considering the uncertainty principle between energy and lifetime operators, determines the time scale over which the radiative transition between two levels occurs. This is found to extend between $10^{-13}-10^{-15} \mathrm{~s}$. These lifetimes are, with a few exceptions, an order of magnitude smaller than other possible de-excitation channels that may occur upon 


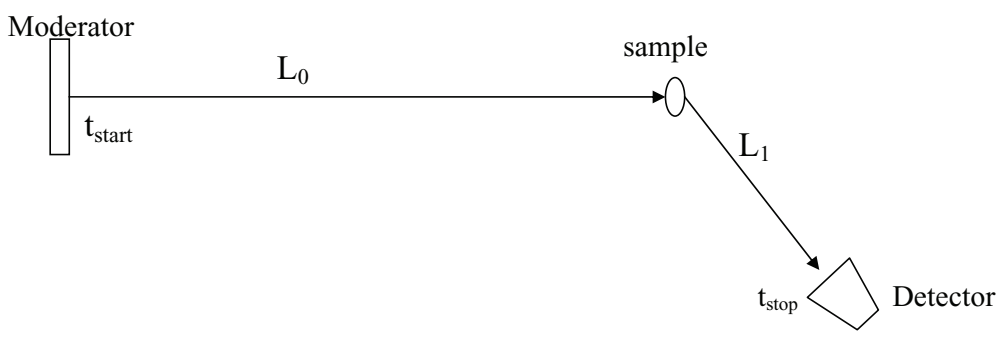

Figure 4. Schematics of the neutron time of flight principle.

slow neutron capture, such as $p$ - or $\alpha$-emission. In a very few light elements $\Gamma_{p}$ and $\Gamma_{\alpha}$ are as large as, or larger than, $\Gamma_{\gamma}$. This is used for example in slow neutron detection using ${ }^{3} \mathrm{He}$ or ${ }^{6} \mathrm{Li}$ or ${ }^{10} \mathrm{~B}$ where secondary charged particle emission is the dominant process (the radiative channel in $n-{ }^{10} \mathrm{~B}$ reaction has a branching ratio of about $93 \%$ ). In heavy fissile nuclei, such as ${ }^{235} \mathrm{U}$, the radiative channel is much less likely than the fission and this physical characteristic might be exploited in neutron detection as well, as for example in thermal and epithermal neutron beam monitoring [26]. The prompt process of radiative capture, the large amount of energy emitted by the gamma rays and the line-structured emission spectra allow to conceive neutron detection approaches based on the detection of the radiative capture gamma rays. These approaches can be used in both elastic and inelastic neutron scattering applications, especially at pulsed spallation neutron sources, as will be discussed in the following sections.

\section{The time of flight technique at pulsed spallation neutron sources}

The advent of spallation neutron sources in the 1980s [5] opened new experimental perspectives in neutron scattering experiments. Being mostly pulsed, it is possible to use the time of flight technique, henceforth named TOF, to reconstruct the kinematics of the scattering process. In what follows we mostly focus on the tests performed at the ISIS spallation neutron source (Rutherford Appleton laboratory, UK) where the counting technique based on radiative capture was extensively refined following the first tests performed in the 1980s. Radiative capture based neutron detectors are now used as detection system for the user program of the VESUVIO electron volt neutron spectrometer [13, 27, 28]. At ISIS, an ion source produces $\mathrm{H}^{-}$ions at $17 \mathrm{keV}$ energy in $200 \mu$ s long pulses to form a beam that is then curved through a $90^{\circ}$ magnet. The $\mathrm{H}^{-}$ions then reach an energy of $35 \mathrm{keV}$ across a DC acceleration gap, and are focused and directed into the radio frequency quadrupole (RFQ) accelerator, operating at $665 \mathrm{keV}$ and 202.5 MHz. Inside the RFQ, four specially shaped electrodes produce an alternating gradient quadrupole electric field for focusing and acceleration. Discrete bunches of $\mathrm{H}^{-}$ ions $4.94 \mathrm{~ns}$ apart are passed into the LINAC, where they are further accelerated up to $70 \mathrm{MeV}$. Before injection into the synchrotron, the electrons of the $\mathrm{H}^{-}$ions are removed by a stripper foil. In the synchrotron, the protons are then accelerated up to $800 \mathrm{MeV}$ and fired onto a (tantalum clad) tungsten target, with a repetition rate of $50 \mathrm{~Hz}$. The neutron pulse produced by spallation is very short (typically few hundreds of nanoseconds) and contains predominantly high energy neutrons. A system of moderators, reflectors and coolers surrounds the spallation target. The purpose of the moderator is to slow down the fast neutrons to the energies required for the neutron instruments (typically between about $1 \mathrm{meV}$ and $100 \mathrm{eV})$, maintaining the time correlation with the proton beam. This allows using the TOF technique for the kinematic reconstruction of the scattering process (i.e. to define energy and wave vector transfer). The total neutron TOF, $t$, is the time a neutron has taken to travel from the moderator to the detector. It can be written as:

$$
t=\frac{L_{0}}{v_{0}}+\frac{L_{1}}{v_{1}}
$$

where $L_{0}$ and $L_{1}$ are the (known) incident and scattering flight paths of the instrument, while $v_{0}$ and $v_{1}$ are the initial and final neutron velocities, The energy transfer is:

$$
\hbar \omega=m \frac{\left(v_{0}^{2}-v_{1}^{2}\right)}{2}
$$

and the momentum transfer:

$$
\hbar q=m\left(v_{0}^{2}+v_{1}^{2}-2 v_{0} v_{1} \cos \vartheta\right)^{1 / 2},
$$

where $\vartheta$ is the scattering angle. Figure 4 represents a schematic layout of a TOF instrument. The TOF technique requires the knowledge of $L_{0}, L_{1}$, the scattering angle $\vartheta$ and the knowledge of either the initial or the final neutron energy $\left(E_{0}\right.$ or $\left.E_{1}\right)$ to reconstruct the kinematics of the scattering process. By knowing the initial energy, the total neutron TOF allows for the measurement of the final one, while the angular position of the detector, $\vartheta$, makes it possible to determine the wave vector transfer, $q$. Each signal is stored in a time channel of a time to digital converter (TDC), its value being the time difference between the initial time of the gate $t_{\text {start }}$ (approximatively corresponding to the instant the neutron leaves the moderator) and the detection instant $\left(t_{\text {stop }}\right)$.

The experimental signal recorded on a typical ISIS beam line is a TOF spectrum, representing the number of counts collected in a time channel of width $\delta t$ centred in $t$. For example, it can be shown that the expression for the count rate per time bin [27, 29] for an inverse geometry spectrometer using a nuclear resonance to define the scattered neutron energy, can be approximated, with good degree of accuracy, by the simple relation $[30,31]$ :

$$
C(t)=A\left(E_{0}, L_{0}, L_{1}\right) \delta t N_{\mathrm{S}} \cdot \zeta \Delta E_{1} \eta_{d}
$$


where $N_{\mathrm{S}}$ is the number of scattering centres in the sample, $\zeta$ the double differential scattering cross section $\frac{\mathrm{d}^{2} \sigma\left(E_{0}, E_{1}, \vartheta\right)}{\mathrm{d} E_{1} \mathrm{~d} \Omega}, \Delta E_{1}$ the total width of the nuclear resonance, $L_{0}, L_{1}$ the primary and the scattering flight paths, respectively and $\eta_{d}$ the overall detection efficiency.

The quantity $A\left(E_{0}, L_{0}, L_{1}\right)$ is given by:

$$
A\left(E_{0}, L_{0}, L_{1}\right)=\frac{\phi\left(E_{0}\right) A_{d} E_{0}^{3 / 2}}{L_{0}^{3} L_{1}^{2} 4 \pi} 2 \sqrt{\frac{2}{m}}
$$

where $\phi\left(E_{0}\right)$ is the flux of the incident neutrons with energy $E_{0}, \frac{A_{d}}{4 \pi L_{1}^{2}}$ is the geometrical acceptance and $m$ the neutron mass.

\section{Theoretical overviews}

\subsection{Deep inelastic neutron scattering}

One of the great advantages of spallation neutron sources is the availability of intense fluxes of epithermal neutrons, typically ranging fom $500 \mathrm{meV}$ to $100 \mathrm{keV}$. These can be used to probe the fast dynamics in molecular systems and/or quantum fluids and solids to investigate the single atom momentum distributions [15]. In this section, deep inelastic neutron scattering (DINS) is briefly introduced for a better understanding of the experimental tests presented in the following. The reader is referred to [15] for a thorough discussion of the technique and detailed description of theoretical issues.

In the DINS regime, the inelastic neutron scattering cross section for unpolarized neutrons is related to the dynamic structure factor $S(\mathbf{q}, \omega)$ via the relation [32-36]:

$$
\frac{\mathrm{d}^{2} \sigma\left(E_{0}, E_{1}, \vartheta\right)}{\mathrm{d} \Omega \mathrm{d} E_{l}}=\hbar^{-1} \sqrt{\frac{E_{1}}{E_{0}}}\left[|b|^{2} S(\mathbf{q}, \omega)+\left(\left|b^{2}\right|-|b|^{2}\right) S_{\mathrm{I}}(\mathbf{q}, \omega)\right],
$$

$b$ and $S_{\mathrm{I}}(q, \hbar \omega)$ being the scattering length of the probed nucleus and the incoherent contribution to the total dynamic structure factor, respectively. At high $q$ values (typically above $20 \AA^{-1}$ ), the scattering is incoherent, meaning that it occurs from a single particle. The typical values of the energy transfer $\hbar \omega$ attainable in DINS experiments range from $1 \mathrm{eV}$ to $100 \mathrm{eV}$, corresponding to a time scale of the order of $10^{-15}-10^{-17} \mathrm{~s}$, which is much shorter than the characteristic times of the high energy collective modes in condensed matter (typically well above $\tau \simeq 10^{-15}$ s). Under these kinematical conditions, the nucleus probed by the neutron recoils freely $[9,15]$. Thus, DINS explores the socalled short-time self-dynamics, and the incoherent and free recoil scattering (resembling that occurring in the Compton scattering of hard x-rays off electrons) manifests in the wellknown impulse approximation (IA). Within the IA, the inelastic neutron scattering cross section in equation $(9)$ is $[9,15]$ :

$$
\frac{\mathrm{d}^{2} \sigma\left(E_{0}, E_{1}, \vartheta\right)}{\mathrm{d} \Omega \mathrm{d} E_{l}}=\hbar^{-1} \sqrt{\frac{E_{1}}{E_{0}}}\left|b^{2}\right| S_{\mathrm{IA}}(\mathbf{q}, \omega),
$$

while the dynamic structure factor is given by [33]:

$$
S_{\mathrm{IA}}(\mathbf{q}, \omega)=\hbar \int n(\mathbf{p}) \delta\left[\hbar \omega-\hbar \omega_{r}-\frac{\mathbf{p} \cdot \hbar \mathbf{q}}{M}\right] \mathrm{d} \mathbf{p},
$$

$n(\mathbf{p})$ being the single particle momentum distribution. Equation (11) establishes that scattering occurs between the neutron and a single particle, while also conserving kinetic energy and momentum of the particle + neutron system. The term $\hbar \omega_{r}=\frac{\hbar^{2} q^{2}}{2 M}$ is the recoil energy, i.e. the kinetic energy the struck particle would have, providing it was stationary before the collision and absorbed all the momentum transferred by the neutron. Within the framework of the IA, $\hbar \omega$ and $q$ are explicitly coupled through the West scaling variable $y$, defined as $[15,32,34]$ :

$$
y=\frac{M}{\hbar^{2} q}\left(\hbar \omega-\hbar \omega_{r}\right)
$$

Equation (11) can then be reduced to the form

$$
S_{\mathrm{IA}}(\mathbf{q}, \omega)=\frac{M}{\hbar q} J(y, \hat{q}),
$$

where

$$
J(y, \hat{q})=\hbar \int n\left(\mathbf{p}^{\prime}\right) \delta\left(\hbar y-\mathbf{p}^{\prime} \cdot \hat{q}\right) \mathrm{d} \mathbf{p}^{\prime}
$$

is the neutron compton profile (NCP), formally defined as the Radon transform of the momentum distribution. The quantity $\hat{q}$ is a unit vector, as $J(y, \hat{q})$ no longer depends on the magnitude of $\mathbf{q}$. The function $J(y, \hat{q}) \mathrm{d} y$ is the probability for an atom to have a momentum parallel to $\hat{q}$ of magnitude between $\hbar y$ and $\hbar(y+\mathrm{d} y)$.

For an isotropic system, the direction $\hat{q}$ is immaterial and equation (14) becomes [15, 37]:

$$
J(y)=2 \pi \hbar \int_{|\hbar y|}^{\infty} p n(p) \mathrm{d} p .
$$

It has to be stressed that in the IA framework, $J(y)$ is symmetrical and centred at $y=0$ and the relation between $n(p)$ and $J(y)$ is $[6,37]$ :

$$
n(p)=-\frac{1}{2 \pi \hbar^{3} y} \cdot\left[\frac{\mathrm{d} J(y)}{\mathrm{d} y}\right]_{\hbar y=p} .
$$

It is worthwhile mentioning that the IA is strictly valid only in the asymptotic double limit $(q, \hbar \omega) \rightarrow \infty$, keeping $y$ constant. For finite values of the energy and wave vector transfers, the longitudinal momentum distribution retains an additional dependence on $q$ which is known as final state effects (FSE). A detailed description of this contribution can be found in references $[35,38,39]$.

\subsection{Thermal neutron diffraction theory}

In this brief section, the theory of diffraction measurements is discussed for time of flight measurements at pulsed spallation neutron sources. Neutron diffraction (ND) is a powerful technique for investigating the crystal structure of materials [40-44]. The diffraction process is described as the deflection of the incident beam by crystal planes $[h k l]$. The well-known Bragg's law:

$$
\lambda=\frac{2 d_{h k l} \sin (\vartheta)}{n}
$$


links together $d_{h k l}$ (the spacing relative to a set of lattice planes), the scattering angle $2 \vartheta$ and the wavelength $\lambda$ of the incident radiation. In order to obtain a full diffraction pattern from a powder sample one of the two parameters $(2 \vartheta$ or $\lambda)$ can be varied leaving the other constant. Diffractometers operating at a spallation source are based on the determination of neutron energy (and hence wavelength) through the TOF technique; in this case Bragg's law can be re-written in terms of TOF $\left(t_{h k l}\right)$ as:

$$
d_{h k l}=\frac{h t_{h k l}}{2 L m_{n} \sin \left(\vartheta_{0}\right)}
$$

where $2 \vartheta_{0}$ is a (fixed) scattering angle, $L$ is the full flight path of the neutrons from the moderator to the detector through the scattering sample and $h$ the Planck's constant. The experimental signal from a TOF diffraction measurement is a pattern of Bragg peaks. Peak positions are directly related to the crystal lattice dimensions and are used to identify phases, structures and/or to infer texture, strain and grain size information through lineshape analysis [45-48].

\section{Development of radiative neutron capture counting techniques}

\subsection{Epithermal neutrons: the resonance detector technique}

As far as epithermal neutron scattering is concerned, since 1986 an extensive research and development activity was carried out at ISIS originally on the eVS and subsequently on the VESUVIO spectrometers [9, 10, 31, 49-66]. Part of this research activity was addressed to develop and enhance the capabilities and effectiveness of the so-called resonance detector spectrometer (RDS) configuration, conceived and tested in the early 1980s [67-73]. As historical examples, figures 5 and 6 show TOF spectra recorded in the RDS configuration at the Dubna (Russia) pulsed reactor IBR-30 [74] with a Ge(Li)${ }^{149} \mathrm{Sm}$ analyser and the RAT spectrometer [72] at the KENS facility (Japan) [75] with a BGO-Ta analyser, respectively.

The main advantages of the RDS configuration are: (i) the counting efficiency is mostly independent of neutron energy [76], unlike techniques that use ${ }^{6} \mathrm{Li}$ or ${ }^{3} \mathrm{He}$ based counters $[57,77]$, (ii) the count rate of the detector is much lower so that it does not saturate and (iii) hybrid data recording approaches, such as the foil cycling technique [78, 79], can be utilized to enhance the resolution and provide environmental gamma background subtraction capability.

An RDS is an inverse geometry instrument where the energy of the scattered neutron is selected by means of resonance analyser and a gamma detector; see figure 7. The RDS counting procedure relies upon two main steps [51, 56, 61, 69, 72]: in the first step, the scattered neutron beam impinges onto the analyser foil which provides the energy analysis by means of $(n, \gamma)$ resonant absorption at a given resonance energy $E_{\mathrm{r}}$. In the second step, the prompt gamma rays are detected and provide the total TOF of the absorbed neutron. It has to be emphasized that the gamma detector is used as a counter: simply provides a trigger to the counting electronics if the signal is



Figure 5. Neutron TOF spectrum from a $\mathrm{TiH}_{2}$ sample and ${ }^{149} \mathrm{Sm}$ analyser foil after $34 \mathrm{~h}$ of data collection. TMA is the TOF given in channel numbers; the channel width is $16 \mu \mathrm{s}$. The peak at $E_{0}$ is produced by elastically scattered neutrons and hence correspond to $E_{\mathrm{r}}=0.873 \mathrm{eV}$, whereas the peaks at $E_{1}$ and $E_{2}$ are due to inelastically scattered neutrons. $E_{1}$ and $E_{2}$ are separated from $E_{\mathrm{r}}$ by $0.14 \mathrm{eV}$ and $0.28 \mathrm{eV}$, respectively. The dashed line indicates the background. Reproduced from [67], Copyright 1981, with permission from Elsevier.



Figure 6. RDS spectrum of polycrystalline graphite measured with a Ta analyser foil and a BGO scintillator: (Upper spectrum) raw data including background and sample holder contribution; (lower spectrum) data after subtraction of the background and sample holder contributions. Reproduced from [72], Copyright 1984, with permission from Elsevier.

above a discrimination threshold that can be set electronically. It is sufficient that one photon among the whole cascade is detected, even by a partial release of its energy in the detector.

The analyser foil has to fulfil some important requirements: (i) the radiative capture cross section has to show isolated and intense resonances in the energy region of interest 


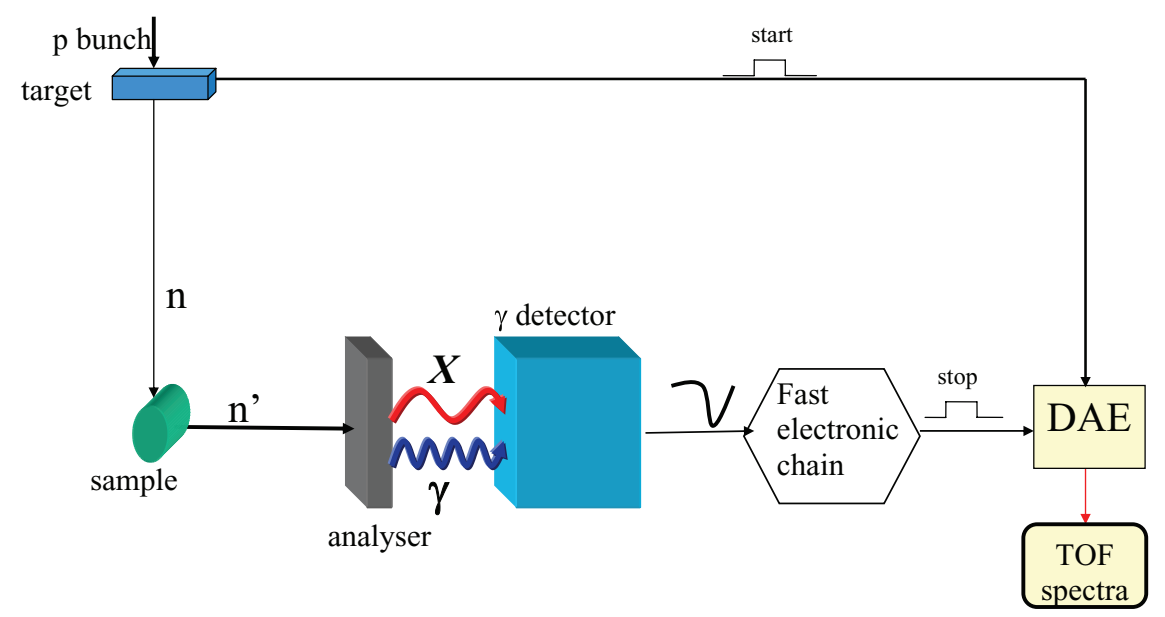

Figure 7. Schematic drawing representing the experimental setup of a resonance detector spectrometer.

Table 1. Main physical parameters of different nuclear resonances that can be utilized in the RDS configuration: $E_{\mathrm{r}}$ is the resonance energy, $\sigma_{0}$ the peak value of the resonance cross section and $\Gamma_{0}$ the half width at half maximum of the resonance.

\begin{tabular}{lccc}
\hline Isotope & $E_{\mathrm{r}}(\mathrm{eV})$ & $\sigma_{0}(\mathrm{~b})$ & $\Gamma_{\gamma}(\mathrm{meV})$ \\
\hline${ }^{113} \mathrm{In}_{49}$ & 14.6 & 9965 & 67 \\
${ }^{139} \mathrm{La}_{57}$ & 72.2 & 5969 & 96 \\
${ }^{150} \mathrm{Sm}_{62}$ & 20.7 & 56207 & 109 \\
${ }^{160} \mathrm{Dy}_{66}$ & 20.5 & 16165 & 124 \\
${ }^{168} \mathrm{Er}_{68}$ & 79.7 & 11203 & 121 \\
${ }^{178} \mathrm{Hf}_{72}$ & 72.6 & 16838 & 112 \\
${ }^{182} \mathrm{~W}_{74}$ & 21.1 & 46800 & 104 \\
${ }^{190} \mathrm{Os}_{76}$ & 91.0 & 6777 & 105 \\
${ }^{197} \mathrm{Au}_{79}$ & 4.96 & 36592 & 60 \\
${ }^{238} \mathrm{U}_{92}$ & 6.67 & 23564 & 25 \\
${ }^{238} \mathrm{U}_{92}$ & 20.8 & 37966 & 34 \\
${ }^{238} \mathrm{U}_{92}$ & 36.6 & 42228 & 57 \\
${ }^{238} \mathrm{U}_{92}$ & 66.0 & 20134 & 48 \\
\hline
\end{tabular}

$\left(E_{\mathrm{r}}=1-100 \mathrm{eV}\right)$, (ii) these resonances should have small widths as compared to $E_{\mathrm{r}}$, (iii) the emitted $\gamma$-ray spectrum should contain lines with appreciable relative intensities at high energies. The first two requirements are important in order to properly select the scattered neutron energy and to ensure a low contribution to the energy component of the resolution function of the spectrometer, respectively. The third requirement allows for selecting gamma rays that provide the best signal to background (S/B) ratio, as it will become clear in the next sections.

In table 1, a number of suitable isotopes that can be used in the RDS configuration are listed together with their main physical parameters. The gamma emission lines of ${ }^{197} \mathrm{Au}$ and ${ }^{238} \mathrm{U}$, the two most widely used isotopes, are shown in figure 8 . The choice of the preferred isotope for the analyser foil depends on the neutron scattering experiment that is performed. The preferred isotope depends for example on the momentum transfer that is assessed, the energy resolution that is required and the data collection time that is allocated for the experiment. The width of the ${ }^{238} \mathrm{U}$ resonances are narrower than the ${ }^{197} \mathrm{Au}$ resonance, giving a better energy resolution, but this narrower width results in less neutrons being absorbed

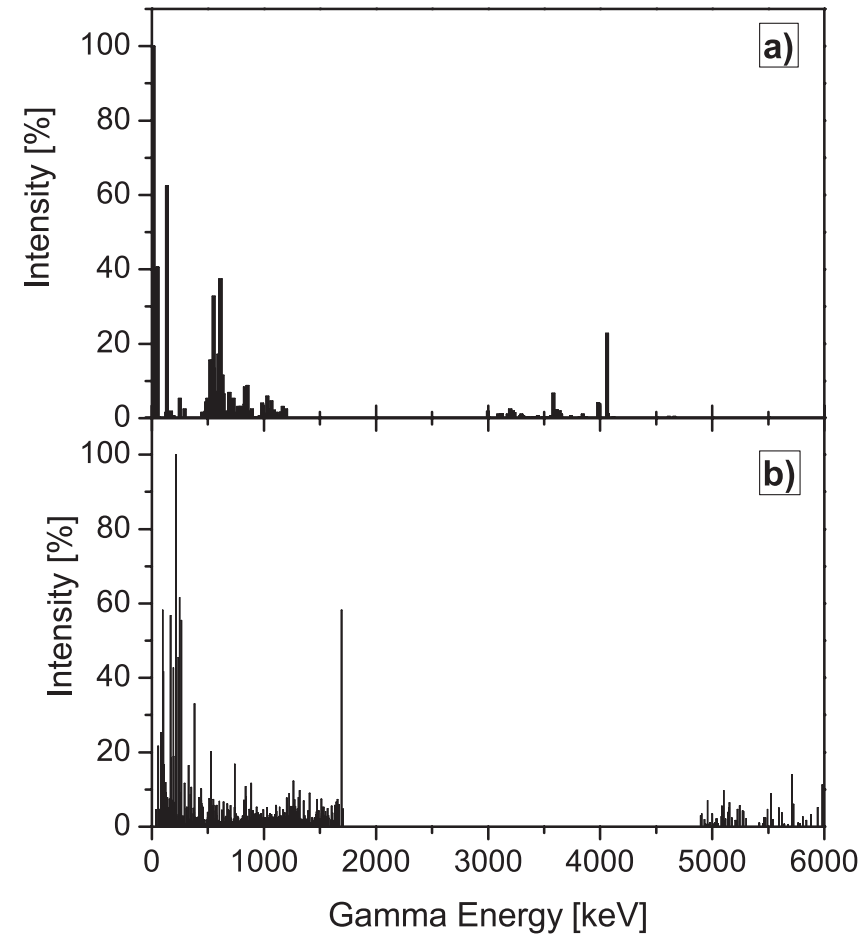

Figure 8. Relative intensity versus $\gamma$ energy for the radiative neutron capture emission of (a) ${ }^{238} \mathrm{U}$ and (b) ${ }^{197} \mathrm{Au}$.

in the analyser foil. For relatively short allocated data collection times, the limited counting statistics with a ${ }^{238} \mathrm{U}$ foil might give less accurate lineshape functions than with a ${ }^{197} \mathrm{Au}$ analyser foil. At ISIS ${ }^{197} \mathrm{Au}$ analyser foils are used for the majority of the experiments.

A further and instructive example of RDS is given in figure 9, where the DINS spectrum in the exchanged energy domain recorded with a $\mathrm{C}_{6} \mathrm{D}_{6}$ liquid scintillator during a DINS measurement on liquid $\mathrm{N}_{2}$ is shown. This measurement was done at the $100 \mathrm{MeV}$ linac neutron source of the National Bureau of Standards (USA) [69]. Another measurement performed at the same facility, in the Filter Difference mode [80], was performed using a BGO and a Germanium detector to assess the effectiveness of narrow energy selection windows in the gamma ray cascade spectrum from 


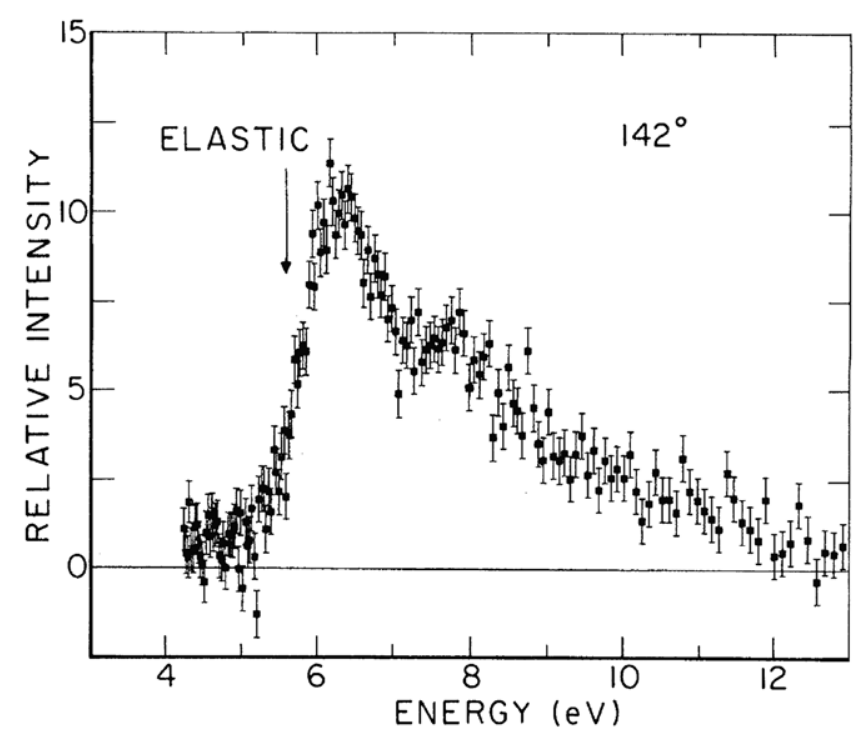

Figure 9. The spectrum of inelastically scattered neutrons from a liquid nitrogen sample using an Au absorber with resonance at $4.9 \mathrm{eV}$. The peak at $7.8 \mathrm{eV}$ arises from an uncorrected background. Reproduced figure with permission from [69]. Copyright 1982 by the American Physical Society.



Figure 10. Neutron time of flight spectrum from an aluminium sample recorded in the RDS configuration using a Bismuth germanate (BGO) gamma detector and an Au analyser foil. In the same plot is also shown the time of flight spectrum from an aluminium sample in the RDS configuration using a high-purity germanium (HPGe) gamma detector and an $\mathrm{Au}$ analyser foil. Data from BGO are a subtraction between analyser in and analyser out data, while HPGe data are a subtraction between analyser in and analyser out and using energy discrimination thresholds on the gamma spectrum from the Au analyser. See [81] for details.

the resonance analyser in achieving an improved S/B ratio. Figure 10 shows the DINS time of flight spectra recorded by means of a BGO scintillation detector without energy selection in the Au gamma ray cascade and a Germanium detector with fine energy selection windows. The reader is referred to [81] for a complete description of the setup. These results show that the S/B doesn't noticeably improve with the use of a fine gamma energy window. The width of the peak is also the same for both data sets. This conclusion can be considered to some extent introductory to the discussion presented in the following sections, where it will be shown how to achieve an
Table 2. Main physical characteristics of $\mathrm{NaI}(\mathrm{Tl})$ scintillator.

\begin{tabular}{ll}
\hline Effective atomic number & 50 \\
Density $\left(\mathrm{g} \mathrm{cm}^{-3}\right)$ & 3.67 \\
Melting point $\left({ }^{\circ} \mathrm{C}\right)$ & 651 \\
Maximum emission wavelength (nm) & 410 \\
Refractive index at $\lambda_{\max }$ & 1.85 \\
Light yield (photons $\left.\mathrm{MeV}^{-1}\right)$ & $410^{4}$ \\
Decay time (ns) & 230 \\
Hygroscopic & Yes \\
\hline
\end{tabular}

optimized S/B in the RDS configurations, highlighting that the energy resolution of the detector is not a key parameter for this application. The following sections will also describe other details of the extensive research and development activity on RDS, carried out at the ISIS spallation neutron source, that have enabled the completion of an operating inverse neutron spectrometer devoted to a scientific user program.

\subsection{Optimising detector type and settings for the resonance detector technique}

The previous section shows results with various types of detectors. For RD detectors to be used permanently on an epithermal neutron spectrometer, detector type, performance and cost all have to be optimised. Hence a systematic research has been done that is described below.

5.2.1. Experiments with sodium iodide scintillation detectors. This section is dedicated to the discussion of the first RDS test, performed on the eVS spectrometer at the ISIS spallation neutron source, with a uranium analyser on the face of a sodium-iodide [ $\mathrm{NaI}(\mathrm{Tl})]$ scintillator [51]. The main characteristics of $\mathrm{NaI}(\mathrm{Tl})$ are highlighted in table 2 . The NaI detector, a cylinder with an $8 \mathrm{~cm}$ diameter and $8 \mathrm{~cm}$ height, was used to detect the high energy component (in the $\mathrm{MeV}$ region) of the gamma ray cascade following resonance radiative capture in uranium; see figure 8 for the energy distribution of the gammas. This RDS test was a DINS measurement on polycrystalline $\mathrm{Pb}\left(100 \mathrm{~cm}^{2}\right.$ area and $1 \mathrm{~mm}$ thickness $)$ [51].

During these measurements data was also recorded with the standard equipment of eVS, which utilizes the same type of analyser foils but use neutron counters based on ${ }^{6} \mathrm{Li}$-glass scintillator. This configuration, also known as resonance filter spectrometer (RFS), is extensively discussed in [80]. For the sake of brevity, we report the results of a $\mathrm{Pb}$ sample, referring the reader to [51] for a more detailed description of the experiment. Figure 11 shows the RDS and RFS TOF spectra recorded by the NaI-U and ${ }^{6} \mathrm{Li}$-glass-U detection systems, respectively. The TOF is shown in a region around the recoil peak of $\mathrm{Pb}$ at the final neutron energy corresponding to the $6.67 \mathrm{eV}{ }^{238} \mathrm{U}$ resonance. The RDS signal is a peak originating from the detection of radiative capture gamma rays by the $\mathrm{NaI}$ detector, while the RFS signal is present as a dip in the TOF spectrum that is produced by resonant neutron absorption in the analyser foil. The large background under the peak in the RDS signal is caused by gamma rays emitted by materials in the experimental area as well as the moderators, reflectors and coolers 




Figure 11. Neutron TOF spectra from a $\mathrm{Pb}$ sample recorded using a $30 \mu \mathrm{m}$ thick uranium foil. The NaI spectrum (top) has a peak corresponding to the $\mathrm{Pb}$ recoil peak at a final neutron energy of $6.67 \mathrm{eV}$. The same resonance gives rise to the absorption dip in the ${ }^{6} \mathrm{Li}$-glass spectrum (bottom). Reproduced from [51]. Copyright 2002, with permission from Elsevier.



Figure 12. Experimental response function $F(y)$ from the NaI scintillator for a $30 \mu \mathrm{m}$ uranium foil and a final neutron energy of $6.67 \mathrm{eV}$. Continuous line is the Voigtian fit. Reproduced from [51]. Copyright 2002, with permission from Elsevier.

surrounding the spallation target; see [52] for more details. The experimental response function $F(y)$, corresponding to the recoil peak recorded in the RDS configuration at $t \simeq 320 \mu \mathrm{s}$ in figure 11 , is plotted in figure 12 . The lineshape analysis on $F(y)$ using a Gaussian fitting model provided a value for the standard deviation $\sigma_{(y, \mathrm{~Pb})}=35.3 \AA^{-1}$ in accordance with the
Table 3. Main physical characteristics of the CZT detector used for DINS experiment on $\mathrm{Pb}$.

\begin{tabular}{ll}
\hline Effective atomic number & $\simeq 50$ \\
$E_{\mathrm{I}}(\mathrm{eV})$ & $\simeq 4.7$ \\
Size $\left(\mathrm{mm}^{3}\right)$ & $5 \times 5 \times 2$ \\
Efficiency at $100 \mathrm{keV}$ & $86.5 \%$ \\
Rise time $(\mathrm{ns})$ & 200 \\
decay time $(\mu \mathrm{s})$ & 200 \\
Energy resolution (FWHM) at $122 \mathrm{keV}(\mathrm{keV})$ & 6 \\
Peak/valley & 2 \\
\hline
\end{tabular}

expectations. This test represented a successful application of the RDS scheme for DINS and demonstrated the viability of the gamma counter approach. However, iodine has a considerable number of resonance peaks in the neutron energy range of interest, see details in [51], also causing the detector to become radioactive during the experiments. An alternative scintillator or gamma detector is therefore required.

\subsubsection{Experiments with a cadmium-zinc-telluride semiconductor} detector. Cadmium-zinc-telluride (CZT) is a ternary semiconductor compound $\mathrm{Cd}_{1-x} \mathrm{Zn}_{x}$ Te with a blending fraction $x$ of CdTe in $\mathrm{ZnTe}[58,82]$ ranging between $5 \%$ and $13 \%$, corresponding to an energy band gap of $1.53 \mathrm{eV}$ and $1.48 \mathrm{eV}$, respectively. The high band-gap values give rise to a low level of leakage-currents, thus allowing room temperature operation [82]. The ability to operate at room temperature allows the construction of compact, low maintenance devices. Table 3 summarizes the main characteristics of one of the CZTs used for the measurements. In order to test the performance of the CZT detectors for the RDS configuration, a DINS experiment was carried out on the VESUVIO spectrometer at ISIS on a $\mathrm{Pb}$ sample, using a $50 \mu \mathrm{m}$ thick ${ }^{238} \mathrm{U}$ analyser foil. The experiment was intended to test the RDS configuration using smaller detectors as compared to the $\mathrm{NaI}(\mathrm{Tl})$ and detecting $\gamma$ energies below $250 \mathrm{keV}$. Figure 13 shows the experimental signal from a $\mathrm{Pb}$ sample in the TOF range $50 \mu \mathrm{s} \leqslant t \leqslant 500 \mu$ s recorded with the CZT detector (figure 13(a)) in the RDS configuration and the corresponding spectrum recorded with the ${ }^{6} \mathrm{Li}$-glass neutron counters placed at a similar scattering angle (figure 13(b)) using the RFS configuration. The S/B ratio is clearly better for the CZT detector with respect to the ${ }^{6} \mathrm{Li}$-glass scintillator detector. This is highlighted in table 4 where the $\mathrm{S} / \mathrm{B}$ is reported for both ${ }^{6} \mathrm{Li}$-glass and CZT detectors at different resonance energies.

A biparametric (TOF versus gamma ray energy) recording system was setup in order to characterise the response of the CZT to the prompt photon emission from both ${ }^{197} \mathrm{Au}$ and ${ }^{238} \mathrm{U}$ analysers. This information can be used to optimize gamma energy regions of interest and hence potentially improve S/B. The analyser foils were placed in the beam to reduce recording time. The reader is referred to $[56,58]$ for a more detailed discussion of the setup. Figure 14 shows the projection of the biparametric data onto the gamma energy axis for two TOF windows, one on-resonance and the other off-resonance, for the first four uranium resonances. The peak at a gamma energy of $133.8 \mathrm{keV}$ is clearly due to resonant neutron capture, so a gamma energy region around this peak will probably 


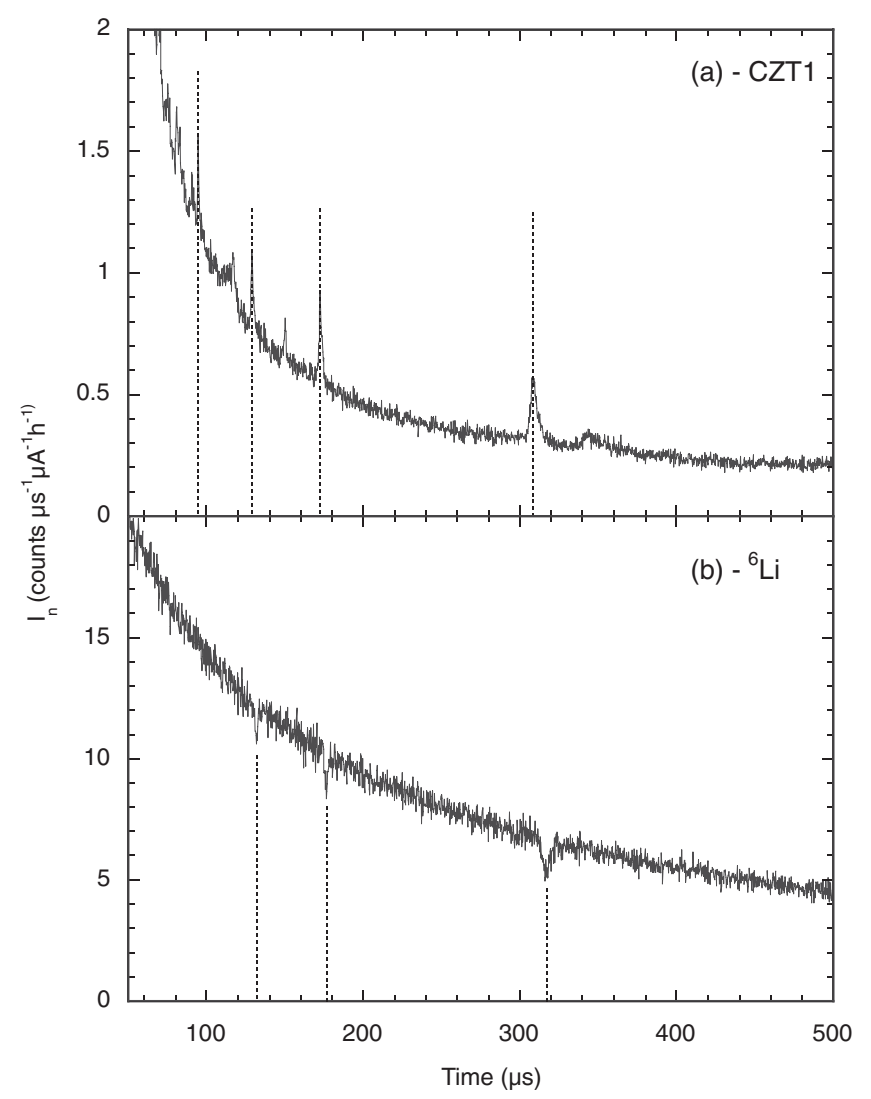

Figure 13. TOF spectra from a $\mathrm{Pb}$ sample recorded with (a) CZT (b) and ${ }^{6} \mathrm{Li}$-glass detectors and ${ }^{238} \mathrm{U}$ analyser foils. Reproduced with permission from [56]. Copyright 2004 IOP Publishing Ltd.

Table 4. Values of the signal-to-background ratio for CZT and ${ }^{6} \mathrm{Li}$-glass detectors, $(\mathrm{S} / \mathrm{B})_{\mathrm{RDS}}$ and $(\mathrm{S} / \mathrm{B})_{\mathrm{RFS}}$ respectively, for the recoil peaks at various final neutron energies, obtained with ${ }^{238} \mathrm{U}$ analyser foils.

\begin{tabular}{lll}
\hline$E_{\mathrm{r}}(\mathrm{eV})$ & $(\mathrm{S} / \mathrm{B})_{\mathrm{RDS}}$ & $(\mathrm{S} / \mathrm{B})_{\mathrm{RFS}}$ \\
\hline 6.67 & 0.44 & 0.14 \\
20.8 & 0.38 & 0.10 \\
36.6 & 0.13 & 0.06 \\
66.0 & 0.14 & - \\
\hline
\end{tabular}

Note: In the case of the highest-energy resonance the ${ }^{6} \mathrm{Li}$-glass (RFS) data were of insufficient statistical quality to allow for a meaningful determination of the S/B. Data from [56].

provide the best $\mathrm{S} / \mathrm{B}$ ratio. Using a gamma energy region of $130.5-135.7 \mathrm{keV}$, figure 15 shows the projection of the biparametric data onto the TOF axis as dashed line. The continuous line is the TOF spectrum obtained without gamma energy discrimination. The $\mathrm{S} / \mathrm{B}$ ratio is indeed better if gamma energy selection is used, but the improvement is surprisingly small and the reduction in signal intensity is very substantial. This is in agreement with the data shown in figure 10, confirming that gamma energy selection is not a viable option and a detector with a good gamma energy resolution is not required.

5.2.3. Experiments with yttrium-aluminium-perovskite scintillators. RDS does not require the use of gamma detectors with a good energy resolution. Scintillator detectors are therefore attractive because of their high density and relatively low

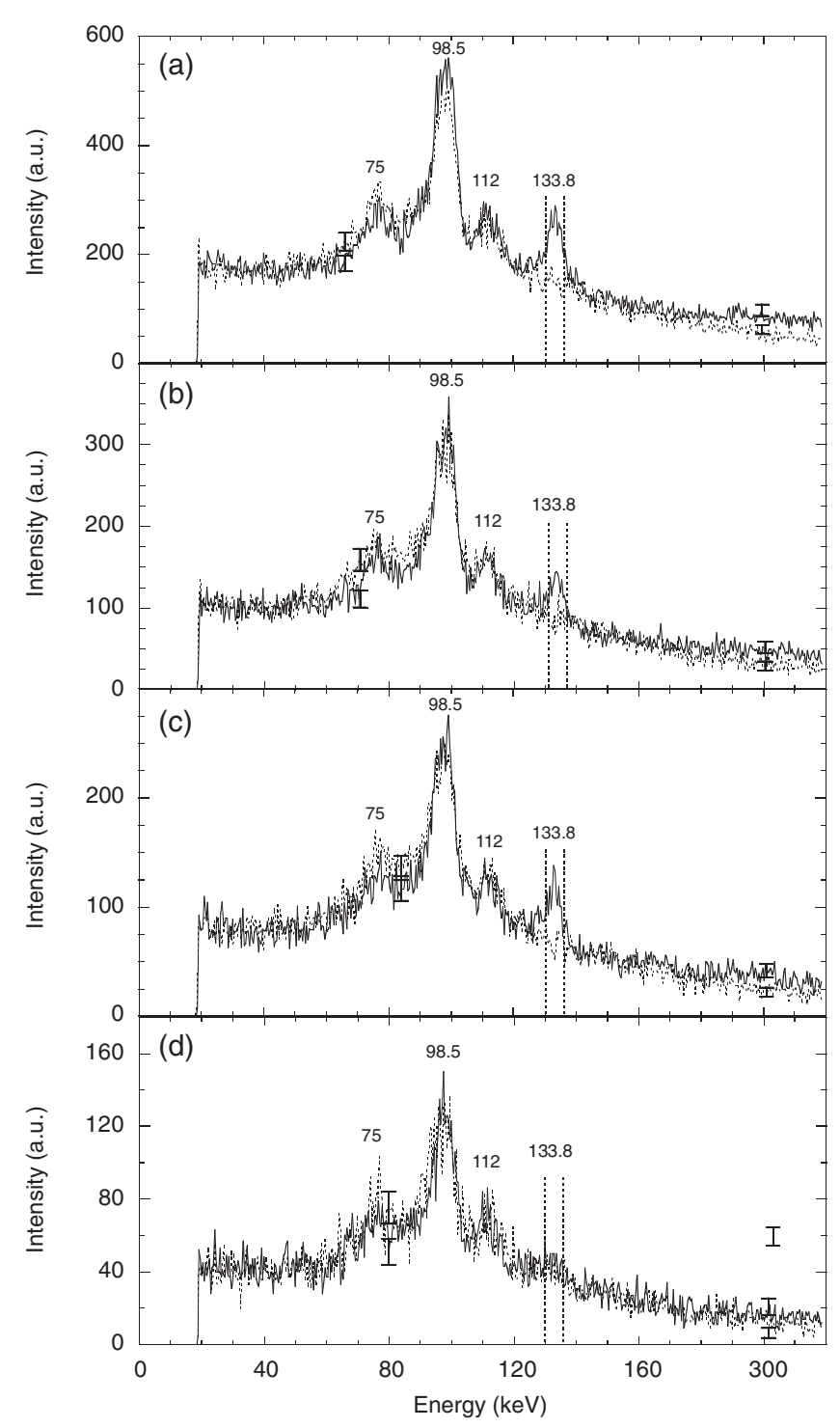

Figure 14. Projection of ${ }^{238} \mathrm{U}$ reduced biparametric data on the energy axis for specified time intervals. The result is the $\gamma$ energy spectrum from neutron capture on ${ }^{238} \mathrm{U}$. In each panel a pair of spectra is shown corresponding to a narrow time window around the neutron resonance (continuous line) and an off-resonance background region (dashed line). The chosen time intervals are: (a) $306.5 \leqslant t \leqslant 312.5 \mu \mathrm{s}$ (resonance) and $198.5 \leqslant t \leqslant 298.5 \mu \mathrm{s}$ (off-resonance); (b) $173.5 \leqslant t \leqslant 176.5 \mu$ s (resonance) and $138.5 \leqslant t \leqslant 168.5 \mu \mathrm{s}$ (off-resonance); (c) $130.5 \leqslant t \leqslant 134.5 \mu \mathrm{s}$ (resonance) and $108.5 \leqslant t \leqslant 128.5 \mu$ s (off-resonance);

(d) $97.5 \leqslant t \leqslant 100.5 \mu \mathrm{s}$ (resonance) and $90.5 \leqslant t \leqslant 96.5 \mu \mathrm{s}$ (off-resonance). The two vertical lines select a $5 \mathrm{keV}$ energy window around the $\gamma$-ray peak at $133.8 \mathrm{keV}$. The statistical error bars are shown for illustrative purpose at the energy values of about 80 and $200 \mathrm{keV}$. Reproduced from [58]. Copyright 2004 with permission from Elsevier.

cost per unit area. The detectors are used as counters and do not require a significant amount of gamma to be absorbed by the photoelectric effect, allowing the use of scintillators with a relatively low atomic number $(\mathrm{Z})$. $\mathrm{YAP}(\mathrm{Ce})$ is therefore an attractive scintillator material because it has no neutron absorption resonances in the energy range of interest for RDS and has favourable scintillation properties. Table 5 lists the main characteristics of $\mathrm{YAP}(\mathrm{Ce})$ scintillator. Figure 16 shows 


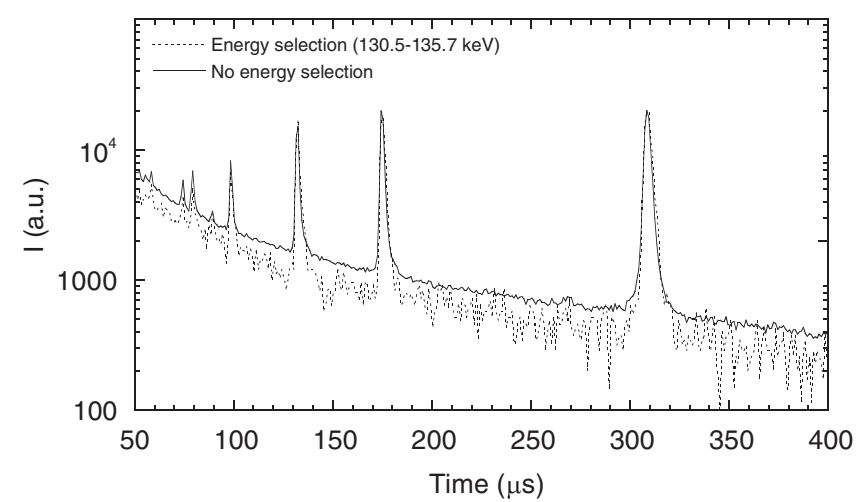

Figure 15. Neutron time-of-flight spectra obtained by projecting the biparametric ${ }^{238} \mathrm{U}$ data onto the time axis for a $130.5-135.7 \mathrm{keV}$ gamma energy region (dashed line) and without any energy selection (continuous line). The spectra have been normalised so that the intensity of the lowest neutron energy resonance is equal to 1 . The S/B ratio is much better than in figure 13 since the analyser foil is in the beam. Reproduced with permission from [56]. Copyright 2004 IOP Publishing Ltd.

Table 5. Main physical characteristics of $\mathrm{YAP}(\mathrm{Ce})$ scintillator.

\begin{tabular}{lc}
\hline Effective atomic number & 36 \\
Density $\left(\mathrm{g} \mathrm{cm}^{-3}\right)$ & 5.55 \\
Maximum emission $\lambda(\mathrm{nm})$ & 350 \\
Refractive index at $\lambda_{\max }$ & 1.94 \\
Light yield (photons $\left.\mathrm{MeV}^{-1}\right)$ & $1.810^{5}$ \\
Decay time (ns) & 27 \\
Hygroscopic & No \\
\hline
\end{tabular}

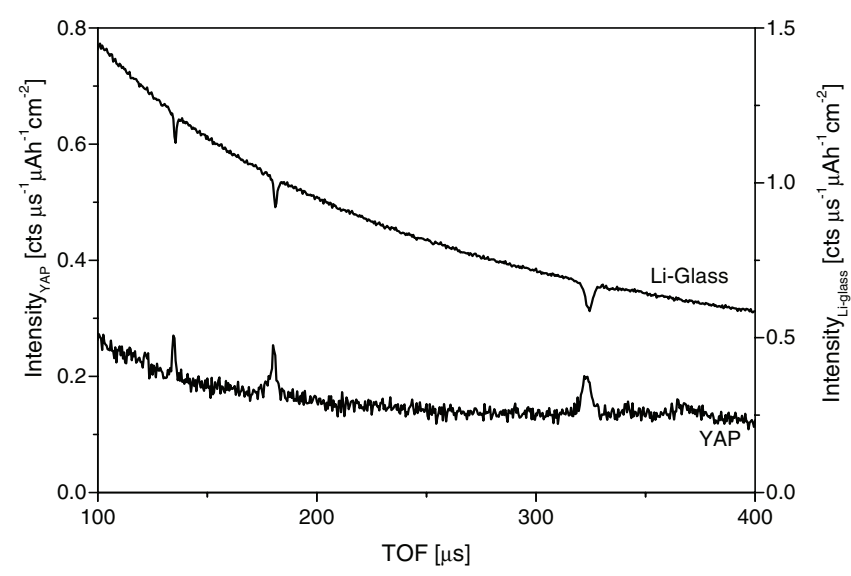

Figure 16. DINS spectra from a $\mathrm{Pb}$ sample and ${ }^{238} \mathrm{U}$ analyser foils recorded with a ${ }^{6} \mathrm{Li}$-glass and YAP detectors placed at the same scattering angle.

a comparison of the DINS spectra from a $\mathrm{Pb}$ sample recorded with a ${ }^{6} \mathrm{Li}$-glass detector and a YAP detector with a gamma discrimination threshold of $40 \mathrm{keV}$. Both detectors were placed at the same scattering angle and use ${ }^{238} \mathrm{U}$ analyser foils. The YAP detector is clearly superior as far as the S/B ratio is concerned. To further optimise the S/B ratio of the YAP detection system, a biparametric acquisition system was setup during DINS experiment on a $\mathrm{Pb}$ sample, as thoroughly discussed in $[77,83]$. These measurements, performed in a realistic setup for the RDS configuration, allowed to determine the best operating conditions for DINS measurements on VESUVIO. A

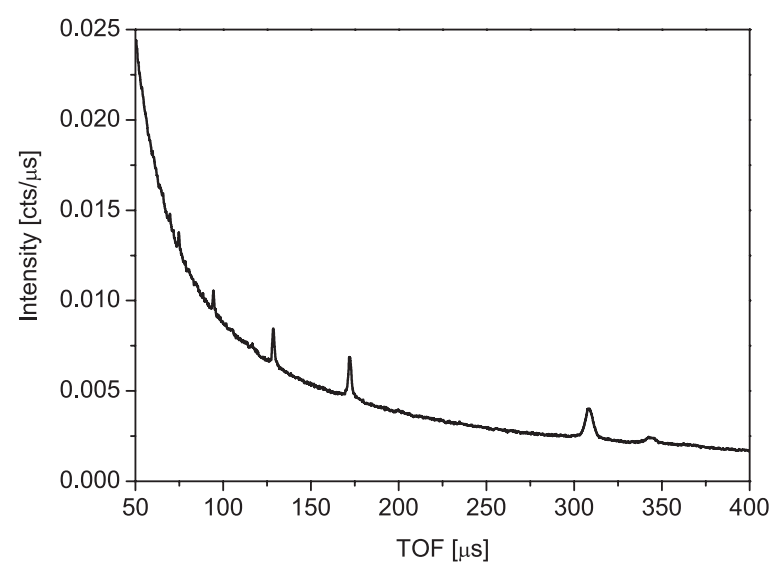

Figure 17. DINS spectrum from a $\mathrm{Pb}$ sample and ${ }^{238} \mathrm{U}$ analyser foils recorded with YAP, utilizing a $40 \mathrm{keV}$ LLD.



Figure 18. DINS spectrum from a $\mathrm{Pb}$ sample and ${ }^{238} \mathrm{U}$ analyser foils recorded with a YAP detector utilizing a $600 \mathrm{keV}$ LLD. The peak at about $350 \mu$ s is the $\mathrm{Al}$ recoil peak, due to the $\mathrm{Al}$ that is present in the sample holder.

thorough characterization of the background radiation present at the spectrometer $[52,84]$ was undertaken to facilitate the analysis of the bi-parametric data. It was found that using a discrimination threshold above circa $600 \mathrm{keV}$, it was possible to maximise the $\mathrm{S} / \mathrm{B}$ and count rate. This can be explained by the suppression of the $480 \mathrm{keV}$ gammas coming from neutron capture on boron that is used as absorbing material in the walls surrounding the spectrometer as well as in the beam dump (see $[52,84])$.

As an example of the impressive improvement of the S/B, figures 17 and 18 show the DINS TOF spectra from a $\mathrm{Pb}$ sample and ${ }^{238} \mathrm{U}$ analyser foils recorded with a Lower Level threshold Discrimination (LLD) of $40 \mathrm{keV}$ and $600 \mathrm{keV}$, respectively. It can be observed that for the peak at $310 \mu \mathrm{s}(\mathrm{Pb}$ recoil peak corresponding to $E_{1}=6.671 \mathrm{eV}$ ) the background is 20 times lower and the peak intensity 2.5 times lower in figure 18 than in figure 17.

A more detailed comparison of the YAP and ${ }^{6} \mathrm{Li}$-glass scintillation detectors is shown in table 6 , where the S/B ratio values for the YAP and ${ }^{6} \mathrm{Li}$-glass detectors are shown for several recoil peaks in the spectra. For all recoil peaks in the spectrum the S/B ratio is better for YAP than for ${ }^{6} \mathrm{Li}$-glass. 
Table 6. List of the signal-to-background (S/B) ratios for the YAP and ${ }^{6} \mathrm{Li}$-glass detectors with ${ }^{238} \mathrm{U}$ analyser foils.

\begin{tabular}{lclll}
\hline$T_{\mathrm{P}}(\mu \mathrm{s})$ & $E_{\mathrm{r}}(\mathrm{eV})$ & $\mathrm{S} / \mathrm{B} \mathrm{YAP}_{\mathrm{L}}$ & $\mathrm{S} / \mathrm{B} \mathrm{YAP}_{\mathrm{H}}$ & $\mathrm{S} / \mathrm{B}^{6} \mathrm{Li}$-glass \\
\hline 310 & 6.67 & 0.60 & 4.00 & 0.12 \\
180 & 20.8 & 0.40 & 6.25 & 0.09 \\
135 & 36.6 & 0.15 & 4.00 & 0.007 \\
90 & 66.0 & 0.13 & 2.72 & \\
70 & 80.3 & 0.09 & 1.24 & \\
55 & 102 & 0.07 & 0.71 & \\
\hline
\end{tabular}

Note: The first column indicate the TOF positions of the $\mathrm{Pb}$ recoil peaks considered, the second column is the corresponding final neutron energy (resonance energy), the third and fourth columns are the observed S/B value for YAP with $\mathrm{LLD}=40$ and $600 \mathrm{keV}$, respectively. The last column is the observed S/B ratio for the ${ }^{6} \mathrm{Li}$-glass detector.

\subsection{Thermal neutron detection}

Thermal neutron detection systems based on radiative capture have been proposed so far, especially for homeland security applications. In this specific context, the use of detection systems relying on the coupling of a neutron absorber such as (mostly) cadmium or gadolinium to a scintillation detector has been investigated. The reader is referred to [85-87] and references therein for a more in depth discussion of this specific topic. The merits of radiative capture based detectors have not yet been fully explored for application in thermal neutron detection at neutron scattering facilities, such as for use in diffractometers. YAP scintillators combined with $\mathrm{Cd}$ converter foils are able to successfully record diffraction data, such as shown by the calibration procedure of the Vesuvio spectrometer. First exploratory tests of the performance of radiative capture based detectors for neutron diffraction have been performed. One of these tests is discussed in more detail in section 6.3. These tests give promising results in the sense that the crystal parameters can be determined with the same accuracy and similar counting efficiency as standard ${ }^{3} \mathrm{He}$ gas tubes, but the observed background under the diffraction peaks is much larger at the moment. A dedicated thermal neutron detector will probably outperform a radiative capture based detector for thermal neutron diffraction measurements in the foreseeable future. However, if epithermal neutron scattering and thermal neutron scattering measurements will be performed with the same detector at the same time, radiative capture based detectors are very good candidate detectors.

\section{Neutron scattering experiments that utilize radiative capture counting techniques}

Neutron scattering experiments have been carried out to verify the performance of radiative capture based detectors for inelastic neutron scattering and thermal neutron diffraction. The reliability of these devices for the RDS configuration is demonstrated in two different inelastic neutron scattering measurements that have been performed on a $\mathrm{H}_{2} \mathrm{O}$ molecular system: (1) a DINS experiment on VESUVIO on liquid bulk water below the critical point, (2) a HINS measurement on the very low angle detector (VLAD) prototype on Ice-Ih



Figure 19. Time of flight spectrum from $\mathrm{H}_{2} \mathrm{O}$ at room temperature and pressure in an $\mathrm{Al}$ container, corrected for background and contributions from the $\mathrm{Al}$ container, using ${ }^{238} \mathrm{U}$ analyser foils. Reproduced with permission from [90]. Copyright 2006 IOP Publishing Ltd.

at $T=270 \mathrm{~K}$. Neutron diffraction measurements on metallic samples, performed at the INES diffractometer [88, 89] at ISIS, are also briefly discussed.

\subsection{DINS measurements on liquid $\mathrm{H}_{2} \mathrm{O}$}

DINS measurements on a liquid $\mathrm{H}_{2} \mathrm{O}$ sample at two different thermodynamic $(P, T)$ conditions, namely $(1 \mathrm{bar}, 293 \mathrm{~K})$ and (100 bar, $423 \mathrm{~K}$ ), have been performed on VESUVIO, using a single YAP detector coupled to a uranium analyser. For the sake of brevity, we report the results of the experiment on (1 bar, $293 \mathrm{~K}$ ) sample in $\mathrm{Al}$ and TiZr sample holders, referring the reader to [90] for a complete and thorough discussion. Figure 19 shows the TOF spectrum from $\mathrm{H}_{2} \mathrm{O}$ in an $\mathrm{Al}$ sample holder after subtraction of the gamma background and correction of the signals from oxygen and aluminium (sample holder). By means of a standard procedure, detailed in [90], the hydrogen peaks in the TOF domain are mapped into the $y$-West scaling variable space to perform the full lineshape analysis that provides the measurement of the mean kinetic energy. Figure 20 shows four experimental response functions measured at two final neutron energies $(20.8$ and $36.6 \mathrm{eV})$ from water in an $\mathrm{Al}$ and a TiZr sample holder.

A comparison was made between the results obtained with YAP-U detection system in RDS configuration and those obtained in the RFS configuration of VESUVIO relying on ${ }^{6} \mathrm{Li}$-glass scintillation detectors. Table 7 reports the results obtained from a data analysis using different models for $J(y)$, namely simple Gaussian, spherically averaged multivariate Gaussian and GaussHermite expanded [91, 92]. These results show that the data of the RDS and RFS configurations are in good agreement.

All information gained with the series of tests discussed in the previous sections and the successful measurements on water, widely and extensively studied with neutron scattering, assessed that the RDS configuration is effective for DINS, achieving better performances as compared to the RFS setup. Moreover, the foil cycling technique (FCT) discussed in section 7.1 has shown the ability to enhance the overall resolution 

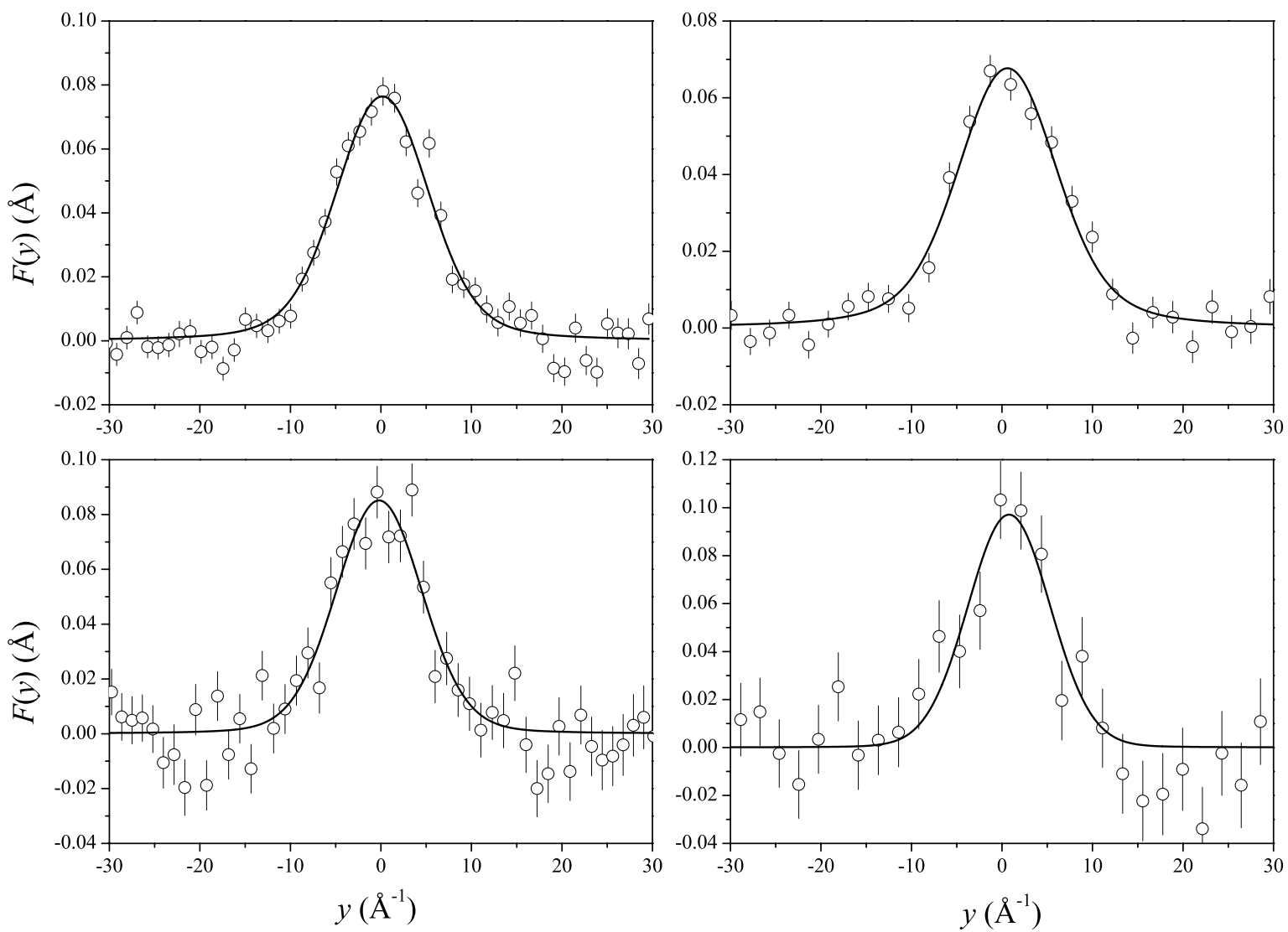

Figure 20. Proton experimental response functions, $F(y)$, and results from the fits obtained using an isotropic Gaussian model for $J(y)$, of $\mathrm{H}_{2} \mathrm{O}$ sample at $(p=1 \mathrm{bar}, T=293 \mathrm{~K})$ in (top left) Al container $\left(E_{1}=20.8 \mathrm{eV}\right)$, (top right) Al container $\left(E_{1}=36.6 \mathrm{eV}\right)$, (bottom left) TiZr container $\left(E_{1}=20.8 \mathrm{eV}\right)$, (bottom right) TiZr container $\left(E_{1}=36.6 \mathrm{eV}\right)$. Reproduced with permission from [90]. Copyright 2006 IOP Publishing Ltd.

Table 7. Values of the proton's means kinetic energy measured in the RDS and RFS configurations for an $\mathrm{H}_{2} \mathrm{O}$ sample at room temperature and pressure.

\begin{tabular}{lll}
\hline Model $J(y)$ & $E_{K}^{\mathrm{RDS}}(\mathrm{meV})$ & $E_{K}^{\mathrm{RFS}}(\mathrm{meV})$ \\
\hline Gauss & $122 \pm 10$ & $124 \pm 3$ \\
Spherical average & $144 \pm 15$ & $147 \pm 8$ \\
Gauss-Hermite & $149 \pm 12$ & $144 \pm 3$ \\
\hline
\end{tabular}

Note: See [90] for details.

of the spectrometer and provide a good subtraction of the gamma background, allowing a more reliable lineshape analysis of the recoil peaks. Since 2007 the VESUVIO spectrometer is operating in the RDS configuration using the FCT for the user research program on the beam line [27].

\subsection{High-energy inelastic neutron scattering on Ice-Ih}

The unique range of exchanged wavevectors, $q$, and energy transfers, $\hbar \omega$, that can be accessed by epithermal neutron scattering experiments makes them an attractive tool for the investigation of condensed matter. The success of DINS experiments provides motivation to extend the application of epithermal neutron scattering. Especially attractive is the extension of the kinematical region of low $\left(1-10 \AA^{-1}\right)$ wavevector transfers, coupled to high energy transfers (typically

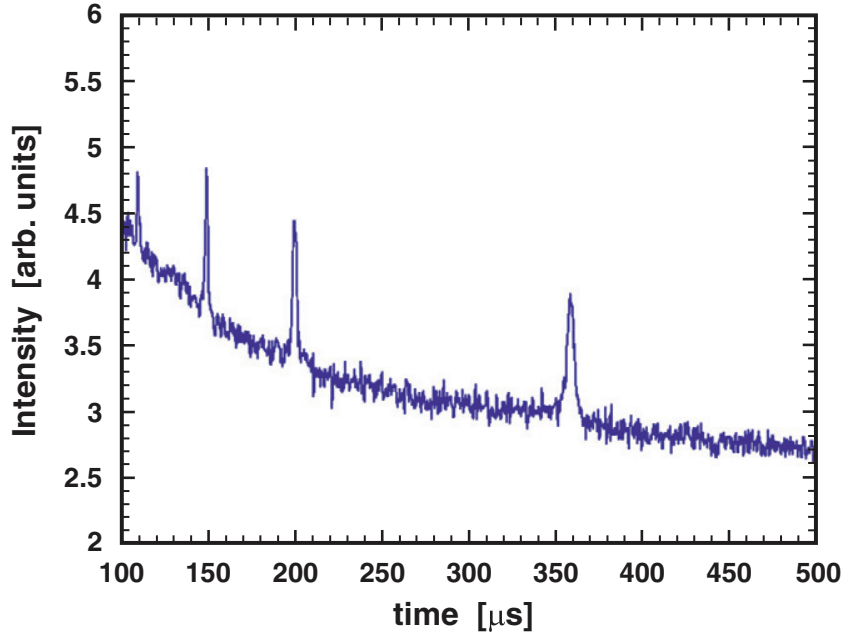

Figure 21. TOF spectra from Ice-Ih, obtained with the VLAD bank and YAP detectors placed at $5^{\circ}$ with ${ }^{238} \mathrm{U}$ analyser foils.

$0.1-10 \mathrm{eV})$ defined as high-energy inelastic neutron scattering (HINS) regime $[61,93]$. The scattering processes in the HINS region are still largely unexplored but would allow experimental investigations such as dispersion relations of high energy excitations in magnetic materials, semiconductors, high lying molecular rotational vibrational states, molecular electronic excitations, and electronic levels in solids [17, 94-96]. 


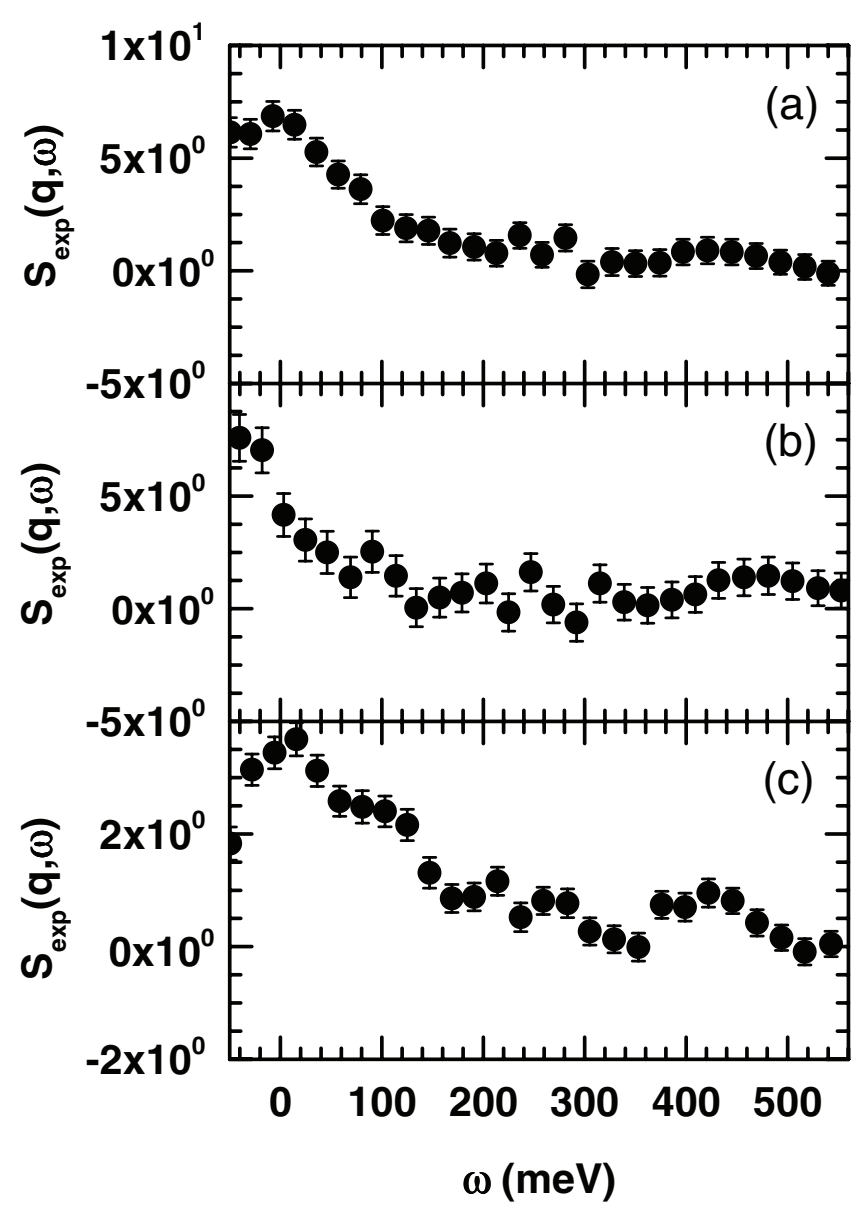

Figure 22. $S(q, \omega)$ from Ice-Ih, obtained by transforming the TOF spectra at angles of: (a) $2.0^{\circ}$ (b) $3.5^{\circ}$ and (c) $5.0^{\circ}$. The $\mathrm{O}-\mathrm{H}$ stretching band is located at approximately $425 \mathrm{meV}$. Reproduced with permission from [61]. Copyright 2004 IOP Publishing Ltd.

In order to develop HINS at spallation neutron sources, a prototype of a low angle eV RDS-based spectrometer was conceived and realized for the VESUVIO spectrometer. The device, called the very low angle detector bank (VLAD), is thoroughly described in [97].

Beyond the scientific interest, HINS measurements on polycrystalline Ice-Ih in the region of the O-H stretching mode provide a very good benchmark for demonstrating the reliability of the RDS detection system in accessing lower $q$ values. The $\mathrm{O}-\mathrm{H}$ stretching mode density of states in polycrystalline Ice-Ih was already been measured, through inelastic neutron scattering [98] on the direct geometry high resolution medium energy chopper spectrometer (HRMECS) at the Argonne National Laboratory, while other authors measured $S(q, \omega)$ and $g(\omega)$ of Ice-Ih at different thermodynamical conditions [99, 100]. The measurements described in [98] have been regarded as the reference term for tests of the RDS detection system. Results, obtained with the VLAD bank and the YAP-U detection system are presented here while a detailed description of the data analysis can be found in a dedicated experimental paper [101]. In figure 21 the TOF spectra from Ice-Ih in an aluminium sample container, for a detector with an angular position of $5^{\circ}$ using a ${ }^{238} \mathrm{U}$ analyser foil, is shown as example. Subtracting the empty

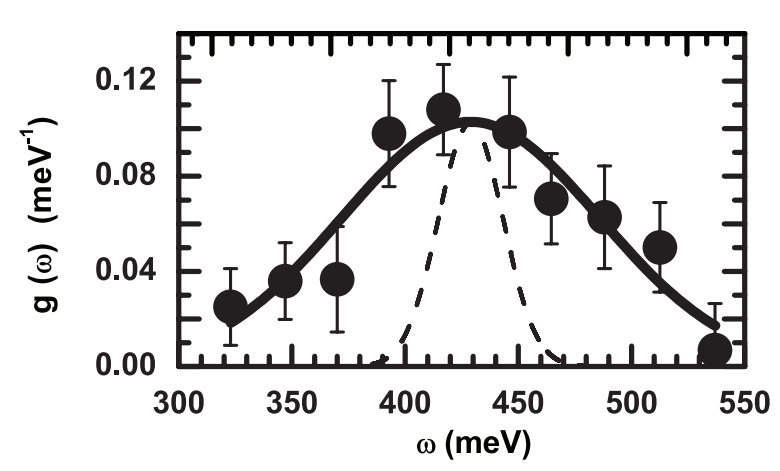

Figure 23. The $\mathrm{O}-\mathrm{H}$ stretching mode energy density of states in the range $300-550 \mathrm{meV}$. The peak is centred around circa $425 \mathrm{meV}$. The dashed line is the $g(\omega)$ obtained by deconvoluting a Gaussian resolution function with $70 \mathrm{meV}$ FWHM from the best fit (full line). Reproduced with permission from [61]. Copyright 2004 IOP Publishing Ltd.

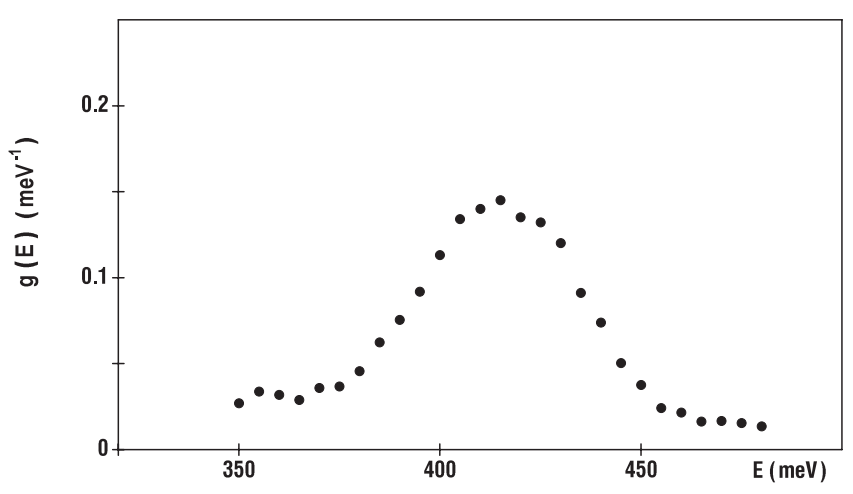

Figure 24. The energy density of states of the O-H stretching mode in polycrystalline Ice-Ih from inelastic neutron scattering measurements on HRMECS at the Argonne National Laboratory. Reproduced with permission from [98]. Copyright 1985 IOP Publishing Ltd.

can spectra from the ones shown in figure 21 and correcting for the residual sample dependent background, the resulting signal spectra at three scattering angles were transformed to obtain the absolute scattering function $S(q, \omega)$ with $q$ varying in the range $2.5-5.5 \AA^{-1}$. This is shown in figure 22 where the peak due to $\mathrm{O}-\mathrm{H}$ stretching is visible on the right-hand side of the main scattering peak (elastic peak at $\omega=0$ ). Following [98], $S(q, \omega)$ was further analysed, bearing in mind that in polycrystalline samples it is related to the density of states $g(\omega)$ through its $q \rightarrow 0$ limit by:

$$
g(\omega)=\lim _{q \rightarrow 0}\left[\frac{S(q, \omega)}{q^{2}} 2 m_{\mathrm{H}} \omega \frac{4 \pi}{\sigma_{\text {inc }}} \frac{1}{n(\omega)+1}\right]
$$

where $n(\omega)$ is the Bose population factor $(\approx 1$ for $T=270 \mathrm{~K})$ and $\sigma_{\text {inc }}$ is the incoherent scattering cross section of hydrogen. With the $q$ values sufficiently close to zero, the shape of the $g(\omega)$ in the O-H stretching energy region, i.e. $350 \mathrm{meV} \leqslant \omega \leqslant$ $450 \mathrm{meV}$, was derived according to equation (19). The resulting $g(\omega)$ values are shown in figure 23. The integral in the stretching region under the $g(\omega)$ curve is:

$$
\int_{350}^{450} g(\omega) \mathrm{d} \omega=9 \pm 2 \text { atoms/cell. }
$$




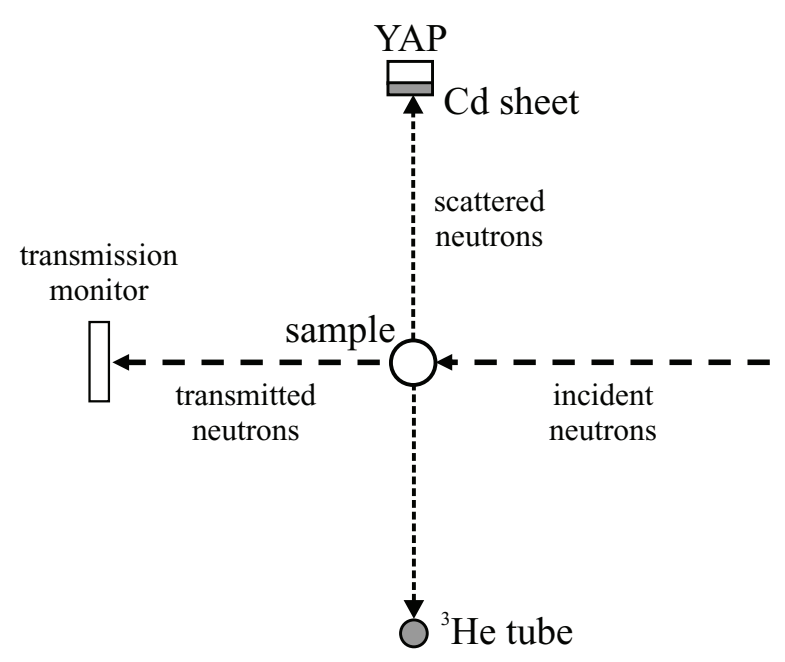

Figure 25. Schematic layout of the INES powder diffractometer at ISIS during the diffraction experiment. The instrument was equipped with a YAP-Cd detector for non-standard diffraction measurements.

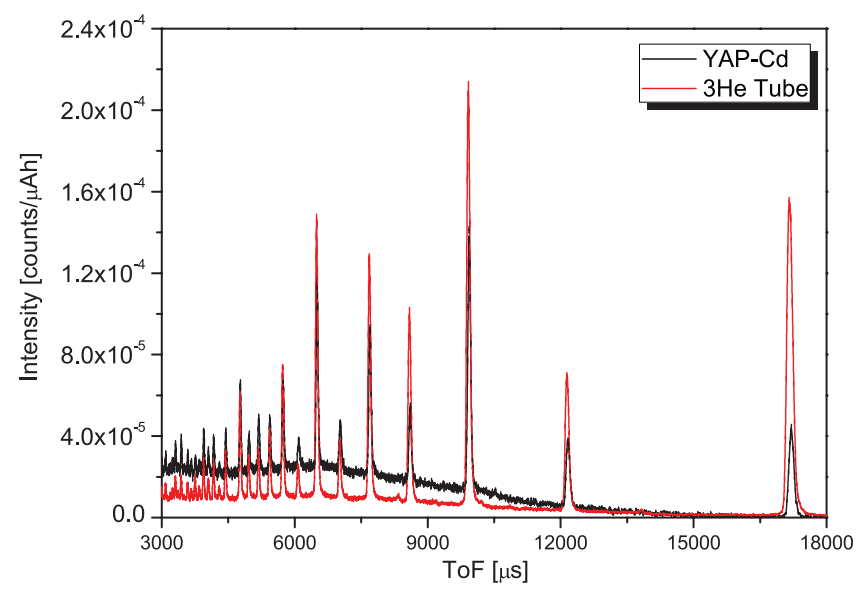

Figure 26. Time of flight diffraction patterns recorded by the YAP-Cd and ${ }^{3} \mathrm{He}$ tube placed at a similar scattering angle in the full thermal neutron energy region. See [102] for more details.

This result is in good agreement with previous measurements on the same system described in [98]. The peak position $(\approx 425 \mathrm{meV})$ is also well reproduced within the experimental error. The latter is due to both statistical uncertainties and to the instrumental resolution (mostly the energy component), which contributes to a large extent to the width of the $g(\omega)$ curve. The effect of the instrumental resolution can be accounted for by deconvoluting its contribution (full width at half maximum $\approx 70 \mathrm{meV}$ ) from a Gaussian fit to the data (full line in figure 23). The width of the resulting Gaussian function (dashed) is in good agreement with earlier measurements [98]. It should be noted that direct geometry (chopper) instruments have an energy resolution which is intrinsically better than inverse geometry instruments. However they are kinematically limited in energy loss to a maximum of about $1 \mathrm{eV}$, whereas inverse geometry instruments are kinematically unlimited. For a quantitative comparison, the $g(\omega)$ obtained by Andreani et al in [98] is shown in figure 24. In that case the $g(\omega)$ is centred around $\approx 417 \mathrm{meV}$, while the value of the integral in equation (20) is 8.1 atoms/cell, which is in good agreement with the result of the HINS experiment on VLAD. It has to be stressed that the HRMECS measurements accessed a lowest $q$ value of about $3-4 \AA^{-1}$, while on VLAD the lowest value of $q$ is about $2 \AA^{-1}$, thanks to the higher final (and thus initial) neutron energies accessed.

\subsection{Use of thermal neutron radiative capture at spallation source instruments}

Thermal neutron radiative capture can be used for detection purposes on $\mathrm{eV}$ instruments like VESUVIO or calibration purposes. In fact, in order to reconstruct the kinematics in DINS and HINS measurements, the angular position of the detector must be determined. Adding a $\mathrm{Cd}$ analyser beyond or after the resonance analyser allows one to record for example the diffraction pattern from sample container or sample (in case this is crystalline). This approach is well described in [90].

Beyond calibration purposes, thermal neutron radiative capture can in principle be used for a complete neutron diffraction experiment. The experimental setup to assess the performance of radiative capture detector for thermal neutron diffraction is schematically shown in figure 25 . The radiative capture detector under investigation was made of a $\mathrm{Cd}$ sheet coupled to a YAP scintillator. The scintillator in turn was connected to a photomultiplier, using a lower level discrimination threshold (LLD) at a photon energy of $600 \mathrm{keV}$ in order to optimize S/B. The Cd sheet acts as a $(n, \gamma)$ converter following the nuclear reaction ${ }^{113} \mathrm{Cd}(n, \gamma){ }^{114} \mathrm{Cd}+7.6$ $\mathrm{MeV}$. The converter sheet was $1 \mathrm{~mm}$ thick, $10 \mathrm{~cm}$ long and $2 \mathrm{~cm}$ wide, the surface area being such to match that of the ${ }^{3} \mathrm{He}$ tubes used on INES. Diffraction patterns, recorded by the YAP and ${ }^{3} \mathrm{He}$ detectors, from an ancient Japanese blade with a high carbon content are reported in figure 26. This data, collected for an integrated proton current of $3350 \mu \mathrm{Ah}$, shows Ferrite and cementite diffraction peaks.

A further analysis was carried out on the diffraction pattern registered with both the ${ }^{3} \mathrm{He}$ and YAP-Cd counters to extract information on phase composition and lattice parameters of the sample. This analysis allows assessing the effectiveness of the YAP-Cd counting device using a direct comparison with a standard detection system. Figure 27 shows experimental data, Rietveld refinement based fit of the peaks and residuals for both ${ }^{3} \mathrm{He}$ and YAP-Cd counters. The weight fraction (wt $\%$ ) of the cementite and ferrite phases determined with the ${ }^{3} \mathrm{He}$ and YAP-Cd counters are compatible within the experimental uncertainties. Further details about this analysis can be found in [102].

\section{Brief overview of the main issues of RDS}

Most of the previously discussed experiments and results have been obtained with single detectors in proof of principle experiments. For resonance radiative capture detectors to be competitive in instruments that operate continuous user-programmes, a couple of issues have to be addressed: energy resolution improvement and gamma background reduction. Indeed, an epithermal neutron spectrometer utilizing neutron detectors 

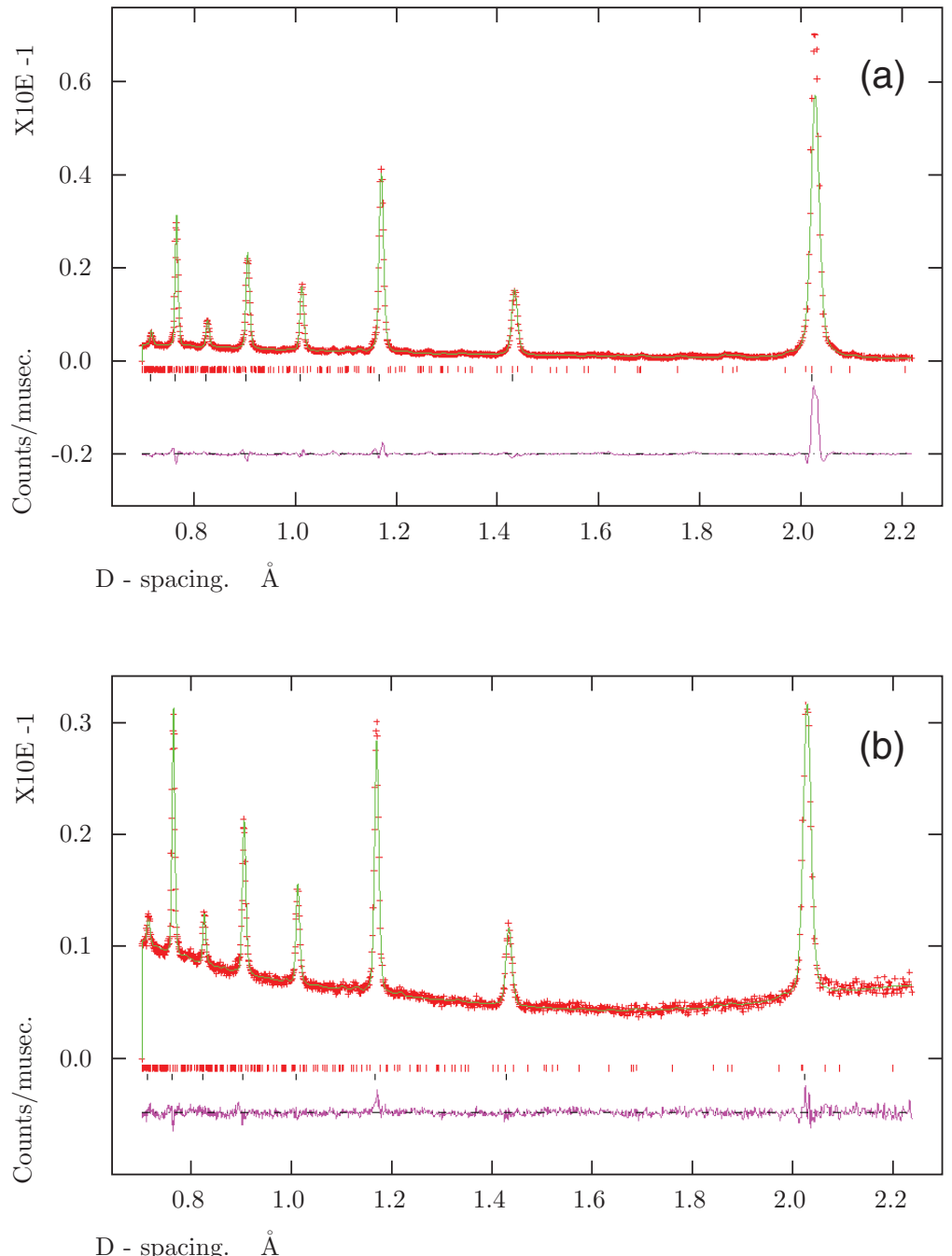

Figure 27. Rietveld analysis of the ancient Japanese blade diffraction pattern recorded by the ${ }^{3} \mathrm{He}$ detector (a) placed at $2 \vartheta=90.565^{\circ}$ and YAP-Cd at $2 \vartheta=90^{\circ}$. In the picture are shown the experimental data points (crosses), the Rietveld refinement fit (continuous line), the reconstructed positions of the Bragg reflections (bars) and the residuals of the fit (lowest continuous line). Reproduced from [102]. Copyright 2011 with permission from Elsevier.



Figure 28. Schematic representation of the setup used in the foil cycling technique.

provides a slightly better resolution than that achievable with a resonant detector spectrometer (RDS) operating in the setup shown in figure 7. As far as gamma background is concerned, section 5 has highlighted that this is a very important issue to be addressed. Both the energy resolution and gamma background drawbacks of RDS can be effectively solved by using the foil 



Figure 29. DINS signals from a $\mathrm{ZrH}_{2}$ sample with an $\mathrm{Au}$ analyser for the 'foil out' and 'foil in' situations are shown on the left. The plot on the right shows the difference signal ('foil out'-'foil in'), illustrating the effective background subtraction obtained with FCT.

cycling technique, as described in the following. Crosstalk, likely to be present in a neutron scattering instrument equipped with an array of gamma detectors, will also be discussed.

\subsection{Foil cycling technique}

A novel signal recording technique named foil cycling technique (FCT) $[78,79]$ provides the possibility to enhance the overall resolution of the spectrometer and obtain a good subtraction of the gamma background. This allows a more reliable lineshape analysis of the recoil peaks. FCT introduces an additional foil, between the sample and detector, that can be cycled in and out of the flight path of the neutrons, see figure 28. The final signal is obtained by subtracting the signal recorded in the 'foil in' position from the signal recorded in the 'foil out' position. A more comprehensive description of the technique is given in [78].

The background arising from environmental gammas is a major issue for RDS signals, as will be obvious from figure 29 and the figures in section 5. Most of this background is slowly varying as a function of TOF and might be removed to some extend by fitting a background function; see for example [90]. The background will be much harder to fit if the sample is contaminated with impurities that have resonance peaks in the TOF region of interest. For radiative capture detectors to be used in routine operation, a simple and reliable background subtraction algorithm is required. For that reason FCT was introduced. As an example of the background subtraction offered by FCT, figure 29 shows TOF spectra recorded with a $\mathrm{ZrH}_{2}$ calibration sample using a gold analyser. The plots on the left show the DINS signals recorded in the 'foil out' and 'foil in' situations. The recoil peaks from $\mathrm{Zr}$ and $\mathrm{H}$ occur at a TOF of $374 \mu$ s and $262 \mu$ s, respectively. The peaks at other TOFs are caused by hafnium contaminations in the $\mathrm{ZrH}_{2}$ sample. The plot on the right of figure 29 shows the signal after taking the difference of the 'foil out' and 'foil in' signals. The large background as well as the peaks arising from the contaminations have been almost completely removed. These peaks, due to resonance neutron absorption in the sample itself, are not affected by the presence of the fixed and cycling analyser

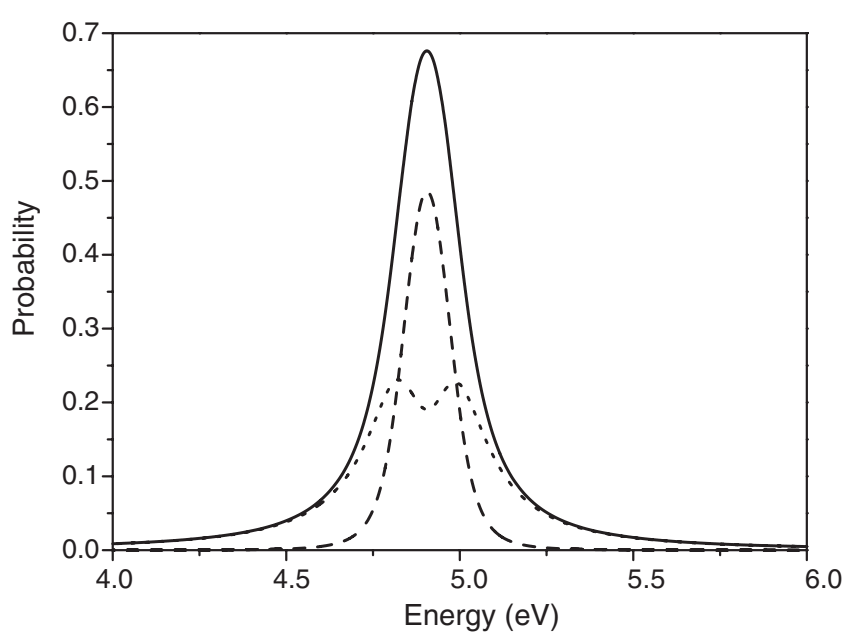

Figure 30. Transfer functions for a single Au analyser foil (continuous line) and for the foil cycling technique (dashed line). The dotted line represents the contribution of 'foil in'. See [79] for details.

foil. Indeed the shielding effect due to Au foil thickness (about $20 \mu \mathrm{m}$ ) is almost negligible, so that in the subtraction procedure these spurious peaks are almost completely cancelled. On the other hand, a small residual is sometimes visible in the signal for very intense background peaks, such as the resonance peak at $285 \mu \mathrm{s}$. Hence a small TOF region might have to be excluded from the analysis, but still FCT allows a much more reliable lineshape analysis than background fitting procedures.

The overall resolution is one of the key parameters of a spectrometer. The resolution is in first instance dependent on the width and shape of the resonance peak of the analyser, see table 1. Notwithstanding, techniques can be used to enhance the resolution function, such as the double difference method (DDM), see [30, 31, 54]. FCT provides a similar improvement in resolution as DDM, as it effectively squares the transfer function $T(E)$; where $T(E)=1-\exp [-N \sigma(E) d]$ with $N$ being the number density of nuclei in the analyser, $\sigma(E)$ the radiative capture cross section and $d$ the thickness of analyser. A thorough description of the transfer function with FCT, and a comparison 


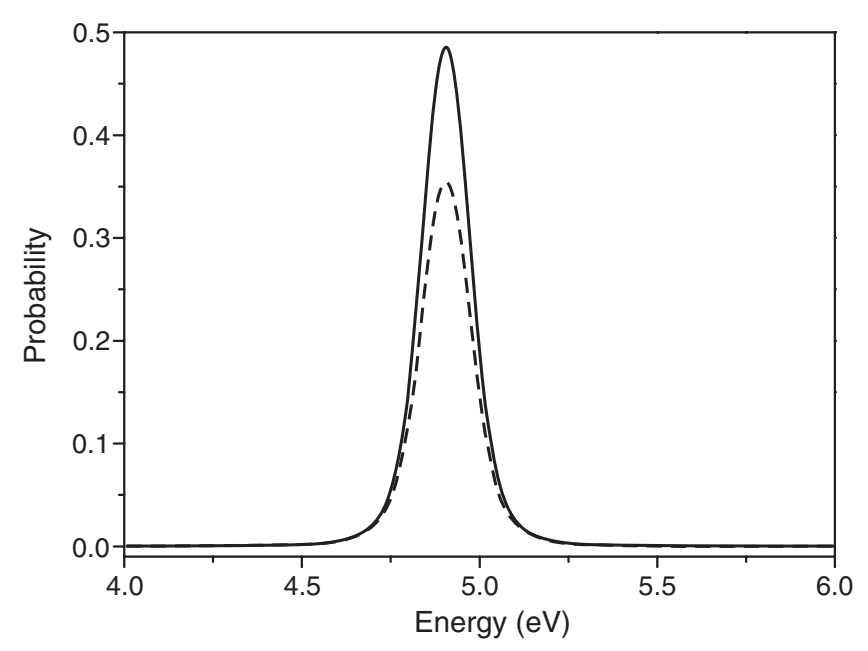

Figure 31. Energy transfer functions obtained through the double difference method (dashed line) and the foil cycling technique (continuous line) for an Au analyser foil. Reproduced from [79]. Copyright 2007 with permission from Elsevier.



Figure 32. Calculation of the gamma signals from DINS on a $\mathrm{Pb}$ sample with Au analyser, utilizing FCT. $P_{\text {out }}-P_{\text {in }}$ is the signal observed in the detector from the analyser when the 'foil in' signal is subtracted from the 'foil out' signal. $S_{\text {in }}$ is the signal observed from the cycling foil in the 'foil in' position, whereas $S_{\text {out }}$ is the signal observed from the cycling foil in the 'foil out' position. $C_{\mathrm{out}}-C_{\mathrm{in}}$ is the total signal, $\left(P_{\text {out }}+S_{\text {out }}\right)-\left(P_{\text {in }}+S_{\text {in }}\right)$, recorded with FCT. Reproduced with permission from [103]. Copyright IOP Publishing Ltd.

of the transfer function of FCT and DDM is given in [79]. To illustrate the improvement in resolution with FCT, figure 30 shows the transfer functions of the standard RDS and FCT. A comparison between the transfer functions of FCT and DDM is shown in figure 31. Besides narrowing the width of the transfer function, FCT also reduces the tails of the transfer function considerably. This is of significant importance for a reliable analysis of DINS signals on light elements heavier than hydrogen. Because of its considerable advantages, FCT has become an essential technique for a resonant detector spectrometer.

\subsection{Residual background}

Although the FCT is very successful in removing environmental gammas, there is still a small background component that will not be removed because it is actually caused by FCT itself. This background is caused by the gamma rays that are detected upon neutron absorption in the cycling foils, and shows up as a dip in the signal to the left side of the recoil

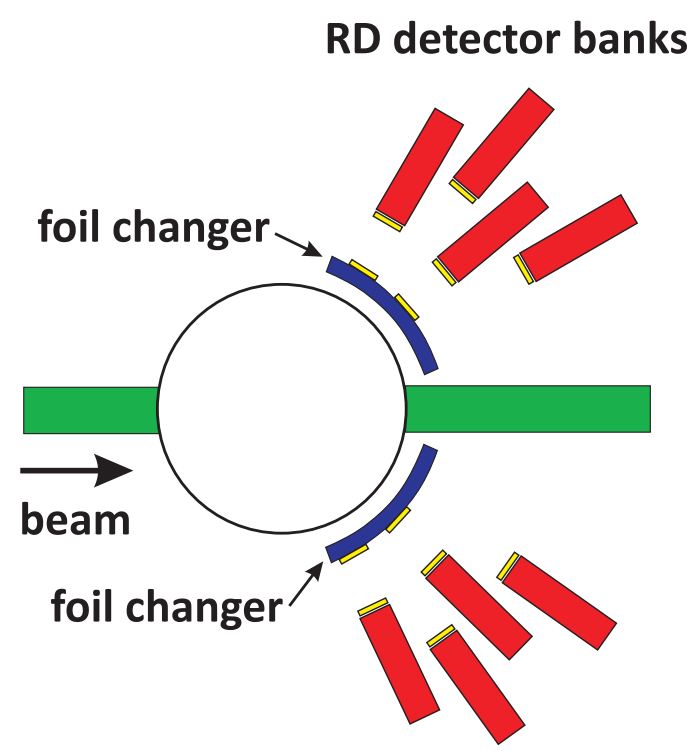

RD detector banks

Figure 33. Schematic representation of the layout of the RD detectors on VESUVIO that reduces crosstalk between detector banks. Each detector bank contains four YAP detectors and associated analyser foils.

peaks. A detailed discussion of this effect is beyond the scope of this paper. Instead, the reader is referred to [103] for an in depth description of this background component and a discussion of how to deal with its effect. As an illustration of how the gamma rays emitted by the cycling foil can distort a DINS FCT signal, figure 32 shows an analytical calculation of the effect of a $20 \mathrm{~cm} \times 20 \mathrm{~cm}^{2}$ Au cycling foil, moving by $20 \mathrm{~cm}$, for a $\mathrm{Pb}$ sample and a sample to detector distance of $55 \mathrm{~cm}$. The residual background is not easily prevented, but, in most cases, can be removed with a simple data processing algorithm [103].

\subsection{Crosstalk reduction}

If the distance between detectors is small, gammas absorbed in the analyser foil in front of a certain detector can actually be registered by another detector. This phenomenon is referred to as crosstalk. Crosstalk can lead to subtle changes in the DINS signal that nevertheless could have a big impact on the accuracy of the results. The effect of crosstalk is not easily shown in DINS signals, as the TOF of the recoil peak of heavy nuclei is independent of the scattering angle and the recoil peak of light nuclei is broad and easily distorted by other effects. A much better indication of the amount of crosstalk can be obtained by examining diffraction data. Crosstalk measurements with YAP detectors and Cd converter foils are subject of present investigation for thermal neutron diffraction experiments on the INES diffractometer at the ISIS spallation source. Crosstalk in these experiments could be reduced by an order of magnitude by staggering the detectors by $25 \mathrm{~cm}$ and maintaining a distance of $15 \mathrm{~cm}$ between detectors in the same plane. A similar crosstalk reduction strategy is followed with the RD detectors on VESUVIO, [103], as shown in figure 33. Results of crosstalk simulations for the very low angle 
detector bank (VLAD) on VESUVIO, [97], are discussed in [104]. Shielding individual detectors is inefficient, but crosstalk can be effectively reduced by maintaining a sufficient distance between detectors at different scattering angles.

\section{Further improvements for RDS}

Sections 5-7 describe the current status at VESUVIO, the only instrument utilizing radiative capture detectors for neutron scattering applications. VESUVIO has been operating a successful user program for years and RD detectors perform well for the current scientific experiments. However, new science requires improved instrumentation and in this section suggestions are given that could improve the performance of a resonance detector spectrometer (RDS).

\subsection{Energy resolution}

A further improvement of energy resolution is desirable for new applications in eV spectroscopy, such as the so-called mass-selective neutron spectroscopy (MANSE) [105]. MANSE is found to be effective in determining the momentum states for multiple masses simultaneously. Indeed, reducing the Doppler broadening of a resonance by cooling the analyser and cycling foils is an option to improve the energy resolution of the spectrometer. However, this is not a simple solution as complex cooling systems are required, especially considering that the cycling foils are moving. Experience has shown that moving cooling systems are likely to create an intolerable background. Moreover, some materials, such as gold that is used at VESUVIO, have resonances with an intrinsically large Breit-Wigner width that will not be reduced by cooling. Cooling the foils is therefore an unattractive option to improve the energy resolution of a RDS. An alternative option could be to apply the double difference method (DMM), see $[30,31,54]$. Although it is possible to combine DDM with the foil cycling technique, calculations have shown that this is as effective in improving the energy resolution as reducing the thickness of the foils and therefore is not attractive either. Presently there does not seem to be a viable method to improve the energy resolution of a RDS, other than changing the isotope of the foils or reducing the thickness of the foils.

\subsection{Sensitivity to background radiation}

An increasing number of scientific experiments investigate complex samples containing both weakly and strongly scattering nuclei. These experiments require detectors with a reduced sensitivity to background gamma rays.

As suggested by Johnson [81] and other authors, the coincidence technique is a candidate that could effectively reduce the sensitivity to gamma rays emitted from the environment, sample and cycling foil. Indeed, this is a very attractive solution as it provides not only an improved signal to background ratio in the measured data, but also offers a reduction in residual background. The reader is referred to section 7.2 for an explanation of the residual background. In section 2.1 it is explained that most nuclei, and especially heavy nuclei, emit a cascade of gamma rays after neutron absorption. Internal conversion electrons are generally also produced. If two or more of these gamma rays or electrons can be detected in two detectors, a coincidence condition can be applied. In practice it is very hard to realise an efficient coincidence set-up, as this requires the solid angle from the analyser foil to both detectors to be large. Such a large solid angle is only possible if the analyser foil is sandwiched between two detectors, in which case one of the detectors is positioned between the sample and analyser foil. Consequently, neutrons will have to pass through the detector before impinging on the analyser foil. Efficient gamma detectors are thick and neutron interaction in such a detector is highly likely, compromising the quality of the measured data. A thin detector between the sample and analyser foil, which detects the internal conversion electrons, is probably a more viable solution. Because of the complications, an effective coincidence set-up for radiative capture detectors used in epithermal neutron spectroscopy has not yet been realised. More research in detector development is therefore required to make the coincidence technique a viable solution for a resonance detector spectrometer.

\subsection{Residual background}

As discussed in section 7.2, the gamma rays from the cycling foils in the foil cycling technique (FCT) cause a residual background that distorts the data. Data analysis algorithms have been developed [103] that are able to successfully correct for these distortions for most scientific experiments. In the future, neutron scattering experiments on compound samples with light elements will become more important and the aforementioned correction introduces unacceptable uncertainties in the results of those scientific experiments. New strategies are therefore required to reduce the distortion in the data caused by the gamma rays emitted by the cycling foils.

Once realised, the coincidence technique will provide a substantial reduction in residual background. Development of the coincidence technique for epithermal neutron spectroscopy will take a considerable time and a successful realisation is uncertain. A more feasible strategy to reduce the residual background is presented here. In this regard, a modification of the foil cycling technique is proposed that significantly reduces the residual background caused by the gamma ray emission of the cycling foil. In the modified FCT, further referred to as enhanced foil cycling technique (eFCT), both the analyser and cycling foils are moved during data collection. A graphical representation of the four states of the foils in eFCT is shown in figure 34 . The philosophy behind eFCT is that when the analyser foil is in the 'analyser foil out' position, the detector only measures the gamma background, which includes the gamma rays emitted by the cycling foil. When the data registered in the 'analyser foil out' states are subtracted from the corresponding data registered in the 'analyser foil in' states, the resulting data will be free of any gamma background; including the background caused by the gamma rays of the cycling foils. As with the standard FCT, the cycling foil is cycled to improve the energy resolution of the spectrometer. 


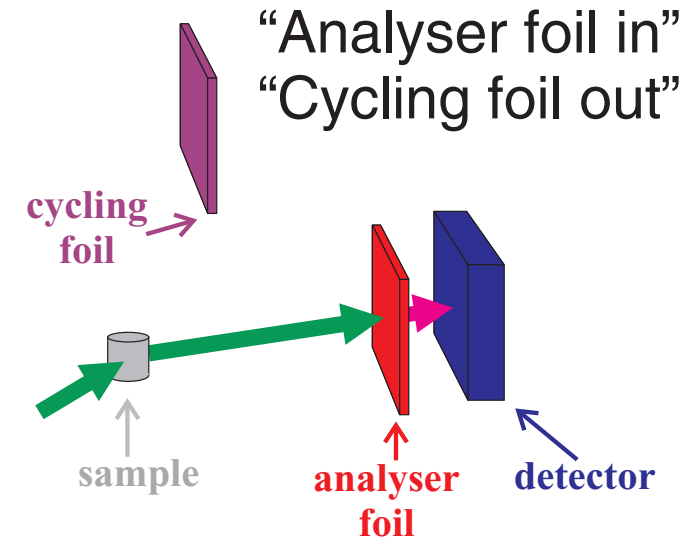

"Analyser foil out" "Cycling foil out"

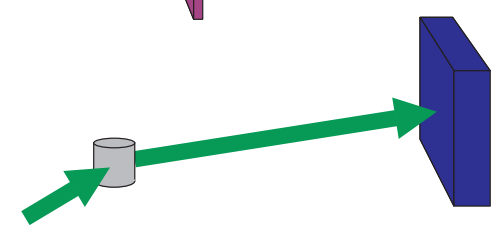

"Analyser foil in"

"Cycling foil in"



Figure 34. Schematic representation of the setup used in the enhanced foil cycling technique (eFCT), where both the analyser foil and cycling foil are moving. In the 'analyser foil out' position, the analyser foil is effectively removed and not shown in the corresponding figures.
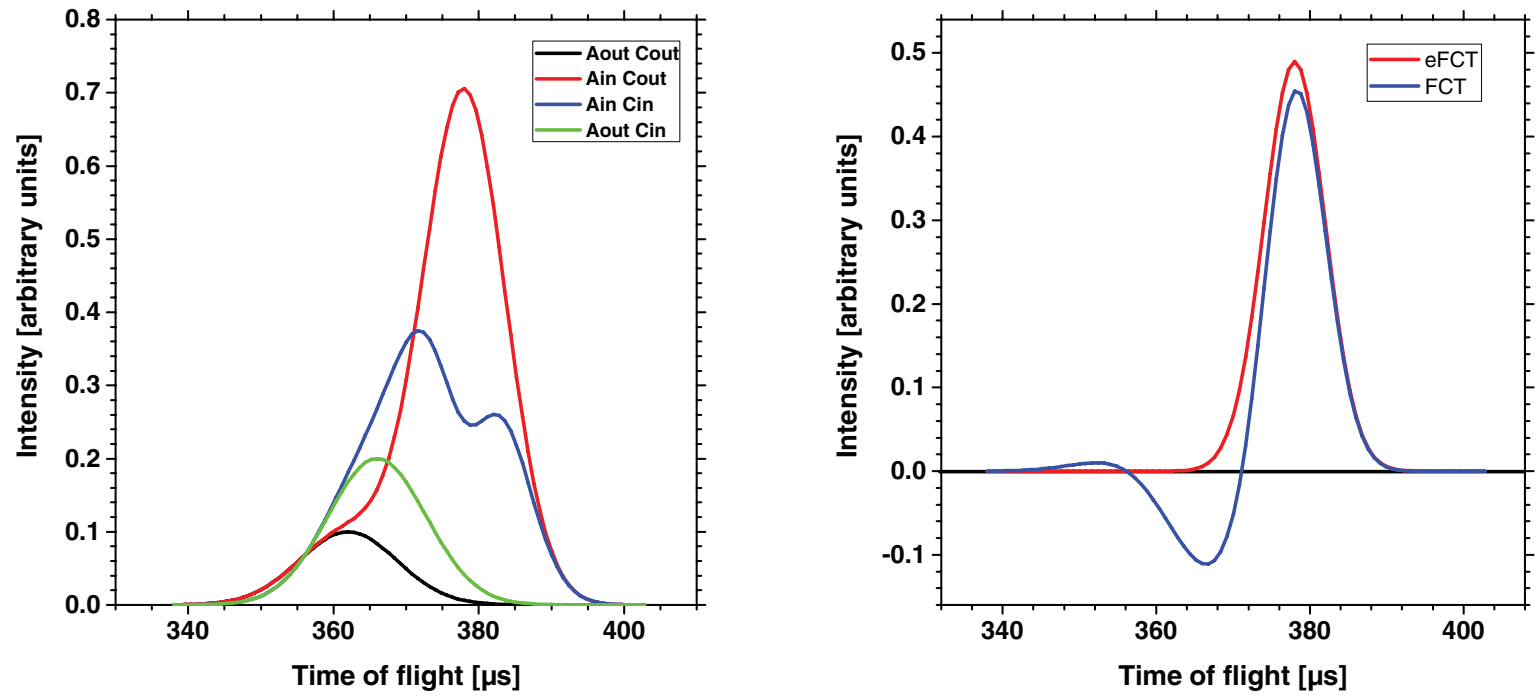

Figure 35. Calculation of the effectiveness of the enhanced foil cycling technique (eFCT). The graph on the left shows the signals measured in each of the four states of an eFCT measurement using ${ }^{238} \mathrm{U}$ analyser and cycling foils. The graph on the right shows a comparison of the final signals of the standard foil cycling technique (FCT) and the proposed eFCT.

In the following text, the data collected in the 'analyser foil in' 'cycling foil out' state is indicated with $A_{\text {in }} C_{\text {out }}$. Likewise, the data collected in the 'analyser foil out' 'cycling foil out' state is indicated with $A_{\text {out }} C_{\text {out }}$. The data collected in the 'analyser foil in' 'cycling foil in' state is indicated with $A_{\mathrm{in}} C_{\mathrm{in}}$. Finally, the data collected in the 'analyser foil out' 'cycling foil in' state is indicated with $A_{\text {out }} C_{\mathrm{in}}$. After proper normalisation of the data, the final signal in the eFCT configuration, $S_{\text {final }}$, is obtained using the formulae:

$$
S_{\text {final }}=\left(A_{\text {in }} C_{\text {out }}-A_{\text {out }} C_{\text {out }}\right)-\left(A_{\text {in }} C_{\text {in }}-A_{\text {out }} C_{\text {in }}\right)
$$

To demonstrate the effectiveness of eFCT, a measurement with a large gamma ray background from the cycling foils is simulated. To make the residual background more severe, the 
time of flight of the gamma rays from the cycling foils is different in the 'cycling foil out' and 'cycling foil in' states. The results of this simulation are shown in figure 35. Unlike the standard FCT, the final signal of eFCT is free of distortions in the form of undershoots and overshoots on the lower time of flight side of the peak. In theory, eFCT will therefore provide a very significant reduction in residual background. In practice a few conditions have to be met that complicate the design of an eFCT set-up. Above all, the detector should not register any gamma rays from the analyser foil in the 'analyser foil out' states. Completely removing the analyser foil is not practical, but moving the analyser foil behind a neutron shield in the 'analyser foil out' states is feasible. The second condition that has to be met is that the mechanics that move the analyser foils scatters neutrons in equal measures in the 'analyser foil out' and 'analyser foil in' positions. A well thought out design of the foil changer will meet this criterion.

A definite disadvantage of eFCT over FCT is that it data collection times will be longer. Since eFCT has four states, compared to two states for FCT, the data collection time in each state is half that of the standard FCT. Additionally, eFCT requires more arithmetic operations on the data, resulting in a higher statistical uncertainty in the results. The increased data collection time will be acceptable for scientific experiments that require a very low residual background. For other experiments, the analyser foils can be kept stationary so that the spectrometer is operating in the standard FCT configuration. eFCT might introduce unforeseen effects that degrade the quality of the recorded data. Further investigations into the effectiveness of the enhanced foil cycling technique are thus required.

\section{Conclusions and future perspectives}

In summary, neutron detection techniques based on radiative capture and their successful application were discussed for both epithermal and thermal neutron scattering applications on pulsed neutron sources.

Resonance radiative capture has been discussed for studies on condensed matter using epithermal neutrons by means of deep inelastic neutron scattering (DINS) and highenergy inelastic neutron scattering (HINS). The main body of this specific research and development activity on the socalled resonance detector spectrometer (RDS) configuration started in the last fifteen years, with the most significant advances made after 2005. This approach, conceived since the first operation of spallation neutron sources, found its mature and effective application on the VESUVIO spectrometer at ISIS; a flagship instrument serving a continuous user-programme for (inelastic) eV neutron spectroscopy measurements. Large amounts of experimental information, gained after several years of extensive experimental activity on radiative capture detectors, demonstrate that the RDS configuration is very effective for neutron spectroscopy at electron volt energies.

Key developments that have contributed to an enhanced performance of this type of spectrometer were directed at improving the resolution and count rate capabilities. These improvements include new methods of energy selection, such as the double difference method and foil cycling technique. On the VESUVIO spectrometer, the use of YAP detectors in conjunction with the foil cycling technique in the forward scattering bank has resulted in a momentum space resolution for proton momentum distribution studies of $\frac{\Delta p}{p}=13 \%$, and counting statistics errors of $1 \%$ at the centre of the Neutron Compton profile and $15 \%$ at $1 / 15$ th of the peak height, respectively. The remaining challenge for a breakthrough in electron volt neutron spectroscopy is improving the energy and angular resolutions. The energy resolution is dominated by the width of the resonances used for energy analysis (approximately $80-100 \mathrm{meV}$ in the best cases), while the angular resolution is mostly determined by the size of the detector and the area of the neutron beam at the sample position.

As far as the radiative capture based detection technique for thermal neutrons is concerned, this approach needs an extensive research and development activity in order to reach the maturity and level of effectiveness achieved for the RDS configuration for eV neutrons. Even though successful diffraction experiments have been demonstrated, crosstalk and signal to background ratio are issues that need specific attention. An improvement of the S/B ratio may be achieved not only by the effective use of energy thresholds, but also by a detailed study and interpretation of the signals coming from the gamma ray cascade from the analysers as compared to the gamma rays from the environmental background.

An emerging powerful application of the radiative capture counting technique is in the field of archaeometry, and archaeology in general. Neutron resonance capture analysis (NRCA) is an established technique for the non-destructive determination of the elemental composition of objects $[106,107]$. Thermal neutron diffraction provides information about the structural composition of objects. Combining both techniques will provide very detailed information about (precious) objects under investigation, but is not well established yet. This multi-tasking approach has been explored by [108] and the ANCIENT CHARM project [109], funded by the New and Emerging Science and Technology activity in the Sixth Framework Programme (FP6) of the European Union. Some successful pioneering archaeometrical measurements have been made [110, 111].

As a final remark, it can be stated that new science requires the development of more powerful and effective instrumentation, in the same way as new instrumentation opens new perspectives for science. Since the first experiments on condensed matter, pioneered by Bertram N. Brockhouse and Clifford G. Shull, considerable progress has been made to make neutron scattering techniques effective for a wider community of users exploiting both elastic and inelastic scattering. In order to make these techniques even more successful, the research and development of instrumentation, especially neutron detectors, is a key issue to be addressed for the neutron sources that are currently under construction. 


\section{Acknowledgments}

Authors acknowledge Consiglio Nazionale delle Ricerche (CNR)-Italy for financial support within the CNR-SERC, CNR-CCLRC and CNR-STFC Agreements (1985-2020) concerning collaboration in scientific research at the ISIS spallation neutron source.

\section{References}

[1] Wollan E O and Shull C G 1948 Phys. Rev. 73830

[2] Zeilinger A, Gähler R, Shull C G, Treimer W and Mampe W 1988 Rev. Mod. Phys. 601067

[3] Brockhouse B N and Hurst D G 1952 Phys. Rev. 88542

[4] Brockhouse B N 1995 Rev. Mod. Phys. 67735

[5] Windsor C G 1981 Pulsed Neutron Scattering (London: Taylor \& Francis)

[6] Hohenberg P C and Platzmann P M 1966 Phys. Rev. 152198

[7] Gol'danskii V I 1957 Sov. Phys._JETP 4604

[8] Ivanov G K and Sayasov Yu S 1967 Sov. Phys. USP. 9670

[9] Gunn J M F, Andreani C and Mayers J 1986 J. Phys. C: Solid State Phys. 19 L835

[10] Andreani C, Baciocco G, Holt R and Mayers J 1989 Nucl. Instrum. Methods A 276297

[11] Mayers J 1993 Phys. Rev. Lett. 711553

[12] Andreani C, Filabozzi A and Pace E 1995 Phys. Rev. B 518854

[13] Senesi R et al 2000 Physica B 276200

[14] Reiter G F, Mayers J and Platzman P 2002 Phys. Rev. Lett. 89135505

[15] Andreani C, Colognesi D, Mayers J, Reiter G F and Senesi R 2005 Adv. Phys. $\mathbf{5 4} 377$

[16] Homouz D, Reiter G, Eckert J, Mayers J and Blinc R 2007 Phys. Rev. Lett. 98115502

[17] Taylor A D, Osborn R, Mcewen K A, Stirling W G, Bowden Z A, Williams W G, Balcar E and Lovesey S W 1988 Phys. Rev. Lett. 611309

[18] Allen D R, Mitchell E W J and Sinclair R N 1980 J. Phys. E 13639

[19] Trohidou K N, Blackman J A and Cooke J F 1991 Phys. Rev. Lett. 672561

[20] Mook H A and Tocchetti D 1979 Phys. Rev. Lett. 432029

[21] Foderaro A 1971 The Elements of Neutron Interaction Theory (Cambridge, MA: MIT Press)

[22] Chrien R E and Kane W R 1979 Neutron Capture Gamma Ray Spectroscopy (New York: Plenum)

[23] Thermal Neutron Capture Gamma-Rays (CapGam) 2016 www.nndc.bnl.gov/capgam/

[24] Breit G and Wigner E 1936 Phys. Rev. 49519

[25] ENDF: Evaluated Nuclear Data File 2016 www-nds.iaea.org/ exfor/endf.htm

[26] Pietropaolo A 2012 Nucl. Instrum. Methods A 67524

[27] Mayers J and Reiter G 2012 Meas. Sci. Technol. 23045902

[28] Seel A G, Krzystyniak M and Fernandez-Alonso F 2014 J. Phys.: Conf. Ser. 571012006

[29] Blostein J J, Dawidowski J and Granada J R 2004 Nucl. Instrum. Methods B 217333

[30] Seeger P A, Taylor A D and Brugger R M 1985 Nucl. Instrum. Methods A 24098

[31] Andreani C, Colognesi D, Degiorgi E, Filabozzi A, Nardone M, Pace E, Pietropaolo A and Senesi R 2003 Nucl. Instrum. Methods A 497535

[32] West G B 1975 Phys. Rep. 18263

[33] Lovesey S W 1986 The Theory Of Neutron Scattering From Condensed Matter (Oxford: Oxford University Press)

[34] Pace E, Salmé G and West G 1991 Phys. Lett. B 273205

[35] Watson G 1996 J. Phys.: Condens. Matter 85955
[36] Scherm R 1972 Ann. Phys. 7349

[37] Andreani C, Filabozzi A, Pace E and Mayers J 1996 Phys. Rev. B 546255

[38] Silver R N 1988 Phys. Rev. B 373794

[39] Silver R N 1989 Phys. Rev. B 394022

[40] Furrer A, Mesot J and Strässle T 2009 Neutron Scattering in Condensed Matter Physics (Singapore: World Scientific)

[41] Mason T E, Gawne T J, Nagler S E, Nestor M B and Carpenter J M 2013 Acta Cryst. A 6937

[42] Siano S, Kockelmann W, Bafile U, Celli M, Iozzo M, Miccio M, Moze O, Pini R, Salimbeni R and Zoppi M 2002 Appl. Phys. A 74 S1139

[43] Festa G, Caroppi P A, Filabozzi A, Andreani C, Arancio M L, Triolo R, Lo Celso F, Benfante V and Imberti S 2008 Meas. Sci. Technol. 19034004

[44] Paradowska A M et al 2012 J. Solid Mech. Mater. Eng. 6408

[45] Arletti R, Cartechini L, Rinaldi R, Giovannini S, Kockelmann W and Cardarelli A 2008 Appl. Phys. A 909

[46] Grazzi F, Bartoli L, Civita F and Zoppi M 2009 Anal. Bioanal. Chem. 3951961

[47] Grazzi F, Bartoli L, Siano S and Zoppi M 2010 Anal. Bioanal. Chem. 3972501

[48] Festa G, Senesi R, Alessandroni M, Andreani C, Vitali G, Porcinai S, Giusti A M, Materna T and Paradowska A 2011 J. Appl. Phys. 109064908

[49] Mayers J, Andreani C and Baciocco G 1989 Phys. Rev. B 392022

[50] Fielding A L and Mayers J 2002 Nucl. Instrum. Methods A 480680

[51] Andreani C, Pietropaolo A, Senesi R, Gorini G, Tardocchi M, Bracco A, Rhodes N and Schooneveld E 2002 Nucl. Instrum. Methods A 481509

[52] Pietropaolo A, Tardocchi M, Schooneveld E M and Senesi R 2006 Nucl. Instrum. Methods A $\mathbf{5 6 8} 826$

[53] Andreani C 2004 Physica B 350231

[54] Mayers J, Tomkinson J, Abdul-Redah T, Stirling W G, Andreani C, Senesi R, Nardone M, Colognesi D and Degiorgi E 2004 Physica B 350 e659

[55] Abdul-Redah T et al 2004 Physica B 350 e837

[56] Andreani C, D'Angelo A, Gorini G, Imberti S, Pietropaolo A, Rhodes N J, Schooneveld E M, Senesi R and Tardocchi M 2004 Appl. Phys. A 78903

[57] Gorini G et al 2004 Nucl. Instrum. Methods A 529293

[58] Tardocchi M, Pietropaolo A, Andreani C, Braxco A, D’Angelo A, Gorini G, Imberti S, Senesi R, Rhodes N J and Schooneveld E M 2004 Nucl. Instrum. Methods A $\mathbf{5 2 6} 477$

[59] Tardocchi M, Pietropaolo A, Senesi R, Andreani C and Gorini G 2004 Nuclear Instrum. Methods A 518259

[60] Tardocchi M, Arnaboldi C, Gorini G, Imberti S, Pessina G, Previtali E, Andreani C, Pietropaolo A and Senesi R 2004 Physica B 350 e853

[61] Andreani C, Pietropaolo A, Senesi R, Gorini G, PerelliCippo E, Tardocchi M, Rhodes N and Schooneveld E M 2004 Appl. Phys. Lett. 855454

[62] Tardocchi M, Pietropaolo A, Andreani C, Gorini G, PerelliCippo E, Rhodes N J, Schooneveld E M and Senesi R 2004 Nucl. Instrum. Methods A 535121

[63] Imberti S, Andreani C, Garbuio V, Gorini G, Pietropaolo A, Senesi R and Tardocchi M 2005 Nucl. Instrum. Methods A 552463

[64] Perelli-Cippo E, Gorini G, Tardocchi M, Cremonesi O, Andreani C, Pietropaolo A, Senesi R, Bowden Z A, Rhodes N J and Schooneveld E M 2005 IEEE Trans. Nucl. Sci. 521092

[65] Tardocchi M, Gorini G, Perelli-Cippo E, Andreani C, Imberti S, Pietropaolo A, Senesi R, Rhodes N J and Schooneveld E M 2006 J. Phys.: Conf. Ser. 41451 
[66] Tardocchi M, Andreani C, Cremonesi O, Gorini G, Perelli-Cippo E, Pietropaolo E, Rhodes N J, Schooneveld E M and Senesi R 2006 Nucl. Phys. B: Proc. Supp. 150421

[67] Cser L, Kroo N, Pacher P, Simkin V and Vasilyeva E 1981 Nucl. Instrum. Methods 179515

[68] Carpenter J M, Watanabe N, Ikeda S, Masuda Y and Sato S 1982 Tests of a resonance detector spectrometer for electron-volt spectroscopy Proc. of the 6th Int. Collaboration on Advanced Neutron Sources pp 266-78

[69] Johnson R G and Bowman C D 1982 Phys. Rev. Lett. 49797

[70] Carpenter J, Watanabe N, Ikeda S, Masuda Y and Sato S 1983 Physica B + C $\mathbf{1 2 0} 126$

[71] Carpenter J and Watanabe N 1983 Nucl. Instrum. Methods 213311

[72] Rauh H and Watanabe N 1984 Nucl. Instrum. Methods A 228147

[73] Crawford R K 1986 The IPNS resonance detector spectrometer Proc. of the 9th Int. Collaboration on Advanced Neutron Sources pp 397-430

[74] IBR-2 Pulsed Reactor: Frank Laboratory of Neutron Physics 2016 http://flnp.jinr.ru/34/

[75] KEK Institute of Materials Structure Science 2016 www2.kek. jp/imss/eng/

[76] Pietropaolo A 2005 PhD Dissertation Università degli Studi di Roma Tor Vergata

[77] Tardocchi M, Gorini G, Pietropaolo A, Andreani C, Senesi R, Rhodes N and Schooneveld E M 2004 Rev. Sci. Instrum. 754880

[78] Schooneveld E M, Mayers J, Rhodes N J, Pietropaolo A, Andreani C, Senesi R, Gorini G, Perelli Cippo E and Tardocchi M 2006 Rev. Sci. Instrum. 77095103

[79] Pietropaolo A, Andreani C, Filabozzi A, Pace E and Senesi R 2007 Nucl. Instrum. Methods A $\mathbf{5 7 0} 498$

[80] Pietropaolo A and Senesi R 2011 Phys. Rep. 50845

[81] Johnson R G 1988 Nucl. Instrum. Methods A 263427

[82] Szeles C and Driver M C 1998 Growth and properties of semiinsulating CdZnTe for radiation detector applications Hard $X$-Ray and Gamma-Ray Detector Physics and Applications (1 July 1998) Proc. SPIE 3446 pp 2-9

[83] Tardocchi M, Pietropaolo A, Andreani C, Gorini G, Imberti S, Perelli-Cippo E, Senesi R, Rhodes N and Schooneveld E M 2006 Nucl. Instrum. Methods A 567337

[84] Pietropaolo A, Perelli Cippo E, Gorini G, Tardocchi M, Schooneveld E M, Andreani C and Senesi R 2009 Nucl. Instrum. Methods A $\mathbf{6 0 8} 121$

[85] Reeder P L 1994 Nucl. Instrum. Methods A 340371

[86] Mitchell D J, Harding L T and Smith K R 2010 IEEE Trans. Nucl. Sci. 572215

[87] Pausch G, Herbacha C-M, Mitchell D, Lentering R and Stein J 2012 Proc. SPIE vol 8382 (Active and Passive Signatures III, 838209 (May 1, 2012))

[88] Grazzi F, Celli M, Siano S and Zoppi M 2007 Nuovo Cimento C 3059

[89] Imberti S et al 2008 Meas. Sci. Technol. 19034003
[90] Pietropaolo A, Andreani C, Filabozzi A, Senesi R, Gorini G, Perelli Cippo E, Tardocchi M, Rhodes N J and Schooneveld E M 2006 J. Instrum. 1 P04001

[91] Andreani C, Degiorgi E, Senesi R, Cilloco F, Colognesi D, Mayers J, Nardone M and Pace E $2001 \mathrm{~J}$. Chem. Phys. 114387

[92] Pantalei C, Pietropaolo A, Senesi R, Imberti S, Andreani C, Mayers J, Burnham C and Reiter G 2008 Phys. Rev. Lett. 100177801

Pietropaolo A, Senesi R, Andreani C, Botti A, Ricci M A and Bruni F 2008 Phys. Rev. Lett. 100127802

Pietropaolo A, Senesi R, Andreani C and Mayers J 2009 Braz. J. Phys. 39318

Senesi R, Flammini D, Romanelli R and Andreani C 2013 Nucl. Instrum. Methods A 704 36-9

Romanelli G, Ceriotti M, Manolopoulos D E, Pantalei C, Senesi R and Andreani C 2013 J. Phys. Chem. Lett. 508 3251-6

[93] Pietropaolo A, Senesi R, Tardocchi M, Andreani C and Gorini G 2004 Physica B 350 e857

[94] Novák P and Diviš M 2007 Phys. Status Solidi B 2443168

[95] Osborn R 1989 Physica B 159151

[96] Agafonov A I 2010 Eur. Phys. J. B 76271

[97] Perelli Cippo E, Gorini G, Tardocchi M, Pietropaolo A, Andreani C, Senesi R, Rhodes N J and Schooneveld E M 2008 Nucl. Instrum. Methods A 589296

[98] Andreani C, Bosi P, Sacchetti F and Loong C K 1985 J. Chem. Phys. $\mathbf{8 3} 750$

[99] Li J C 1996 J. Chem. Phys. 1056733

[100] Kolesnikov A I, Li J C, Parker S F, Eccleston R S and Loong C K 1999 Phys. Rev. B 593569

[101] Perelli Cippo E, Gorini G, Tardocchi M, Andreani C, Pietropaolo A, Senesi R, Rhodes N J and Schooneveld E M 2006 Appl. Phys. A 83453

[102] Festa G, Pietropaolo A, Grazzi F, Barzagli E, Scherillo A and Schooneveld E M 2011 Nucl. Instrum. Methods A 654373

[103] Mayers J 2011 Meas. Sci. Technol. 22015903

[104] Perelli Cippo E, Gorini G, Tardocchi M, Andreani C, Pietropaolo A, Senesi R, Rhodes N J and Schooneveld E M 2008 Meas. Sci. Technol. 19047001

[105] Seel A G, Ceriotti M, Edwards P P and Mayers J 2012 J. Phys.: Condens. Matter 24365401

[106] Postma H, Perego R C, Schillebeeckx P, Siegler P and Borella A 2007 J. Radioanal. Nucl. Chem. 27195

[107] Schillebeeckx P et al 2012 J. Instrum. 7 C03009

[108] Pietropaolo A, Festa G, Grazzi F, Barzagli E, Scherillo A, Schooneveld E M and Civita F 2011 Europhys. Lett. 9548007

[109] Gorini G 2007 Ancient charm: a research project for neutron based investigation of cultural-heritage objects Il Nuovo Cimento C 30 47-58

[110] Postma H, Amkreutz L, Borella A, Clarijs M, Kamermans H, Kockelmann W, Paradowska A, Schillebeeckx P and Visser D 2010 J. Radioanal. Nucl. Chem. 283641

[111] Festa G, Pietropaolo A, Grazzi F, Sutton L F, Scherillo A, Bognetti L, Bini A, Barzagli E, Schooneveld E and Andreani C 2013 Meas. Sci. Technol. 24095601 